\title{
Conserved human effector Treg signature is reflected in transcriptomic and epigenetic landscape
}

Authors: Gerdien Mijnheer, ${ }^{1 \dagger}$ Lisanne Lutter, ${ }^{1 \dagger}$ Michal Mokry, ${ }^{1,2,3}$ Marlot van der Wal, ${ }^{1}$ Veerle Fleskens, ${ }^{4}$ Rianne Scholman, ${ }^{1}$ Aridaman Pandit, ${ }^{1}$ Weiyang Tao, ${ }^{1}$ Mark Wekking, ${ }^{3}$ Stephin Vervoort, ${ }^{1,5}$ Ceri Roberts, ${ }^{4}$ Alessandra Petrelli, ${ }^{1}$ Janneke G.C. Peeters, ${ }^{1}$ Marthe Knijff, ${ }^{1}$ Sytze de Roock, ${ }^{1}$ Sebastiaan Vastert, ${ }^{1}$ Leonie S. Taams, ${ }^{4}$ Jorg van Loosdregt, ${ }^{1,5 \dagger}$ Femke van Wijk ${ }^{1 \dagger^{*}}$ $†$ Shared position

\section{Affiliations:}

${ }^{1}$ Center for Translational Immunology, Pediatric Immunology \& Rheumatology, Wilhelmina Children's Hospital, University Medical Center Utrecht, Utrecht University, Utrecht 3508 AB, the Netherlands.

${ }^{2}$ Regenerative Medicine Center Utrecht, Department of Pediatrics, University Medical Center Utrecht, Utrecht 3508 AB, The Netherlands.

${ }^{3}$ Epigenomics facility, University Medical Center Utrecht, Utrecht 3508 AB, The Netherlands.

${ }^{4}$ Centre for Inflammation Biology and Cancer Immunology, School of Immunology \& Microbial Sciences, King's College London, London SE1 1UL, UK.

${ }^{5}$ Regenerative Medicine Center Utrecht, Center for Molecular Medicine, University Medical Center Utrecht, Utrecht 3508 AB, The Netherlands.

*Corresponding author:

Femke van Wijk, PhD

Center for Translational Immunology, Wilhelmina Children's Hospital, University Medical Center Utrecht, Lundlaan 6, 3584 EA Utrecht. Tel +31 887554275

Email: f.vanwijk@umcutrecht.nl 


\begin{abstract}
Treg are critical regulators of immune homeostasis, and increasing evidence demonstrates that environment-driven Treg differentiation into effector (e)Treg is crucial for optimal functioning. However, human Treg programming under inflammatory conditions remains poorly understood. Here, we combine transcriptional and epigenetic profiling to identify the human eTreg core signature. Functional autoimmune inflammation-derived Treg display a unique transcriptional profile characterized by upregulation of both a core Treg (FOXP3, CTLA4, TIGIT) and effector program (GITR, BLIMP-1, BATF). We identified a specific human eTreg signature that includes the vitamin D receptor (VDR) as predicted key-regulator in eTreg differentiation. H3K27ac/H3K4me1 occupancy revealed pronounced changes in the (super)enhancer landscape, including enrichment of the binding motif for VDR and BATF. The observed Treg profile showed striking overlap with tumor-infiltrating Treg. Our data demonstrate that human inflammation-derived Treg acquire a specific eTreg profile guided by epigenetic changes. The core eTreg profile is conserved, and fine-tuned by environment-specific adaptations.
\end{abstract}




\section{Introduction}

Forkhead box $\mathrm{P} 3$-expressing $\left(\mathrm{FOXP}^{+}\right)$Treg are key players to control aberrant immune responses. Mutations in the FOXP3 gene lead to severe autoimmunity and inflammation in both mice and men $^{1,2}$. Because of their potential for clinical applications, Treg have been intensively studied over the last decades. Despite this effort there is still a fundamental gap in knowledge, especially regarding the gene expression profiles and epigenetic regulation of human Treg in inflammatory settings.

There is accumulating evidence from mouse models that specific environments such as inflammation or non-lymphoid tissues can induce further differentiation/adaptation into specialized activated Treg subsets, also referred to as effector (e)Treg ${ }^{3,4}$ (reviewed in ref. ${ }^{5}$ ). Characteristic for eTreg appears to be the maintenance of FOXP3 expression, increased expression of several molecules related to their function such as ICOS, CTLA4 and TIGIT, and adaptation to the local environment. For example, recent studies in mice show that in visceral adipose tissue Treg play a dominant role in metabolic homeostasis ${ }^{6}$, whereas Treg in skin and gut can promote wound repair and are involved in local stem cell maintenance ${ }^{7,8}$. It remains poorly understood how this specialization in tissues is acquired and regulated at a transcriptional and epigenetic level.

In mice, eTreg have been demonstrated to be crucial in specific inflammatory settings. Interestingly, inflammatory signals can induce the stable upregulation of typical Th cell transcription factors such as T-bet, allowing the Treg to migrate to the site of inflammation ${ }^{9}$. Moreover, co-expression of T-bet and Foxp3 is essential to prevent severe Th1 autoimmunity ${ }^{10}$. Studies using transgenic mice have eminently contributed to the knowledge in this field, but how 
this can be translated to humans remains to be elucidated. Because of the interest in the use of Treg for therapeutic purposes in a variety of human diseases it is highly relevant to gain insight in human eTreg programming in inflammatory settings.

Also in humans there is evidence of environment-induced adaptation of human (e)Treg ${ }^{4,7,11}$ (reviewed in ref. ${ }^{12}$ ) including tumor-infiltrating Treg signatures ${ }^{13-15}$. Pertinent issues that remain to be addressed are how diverse and stable human eTreg programming is in the inflammatory environment, and whether this is regulated at an epigenetic level.

Here, we investigated the gene expression profile and active enhancer landscape of human Treg in an autoimmune-associated inflammatory environment, i.e. synovial fluid (SF) from inflamed joints of Juvenile Idiopathic Arthritis (JIA) and Rheumatoid Arthritis (RA) patients. Transcriptome analysis of SF Treg revealed a classical Treg signature as well as strongly upregulated markers including ICOS, BATF, T-bet, IL-12r 32 and Blimp-1, indicating eTreg differentiation and adaptation to the inflammatory environment. Data-driven network analysis further revealed the vitamin $\mathrm{D}_{3}$ receptor (VDR) as a predicted key-regulator of eTreg differentiation. Importantly, H3K27ac and H3K4me1 ChIP-seq showed that this dual programming was imprinted at an epigenetic level. These eTreg demonstrated normal suppressive function and enhanced IL-2 signaling. Finally, a substantial overlap between the SF Treg signature and recently published human tumor-infiltrating Treg signatures were found. These findings indicate that human eTreg programming is epigenetically imprinted and may be commonly induced in inflammatory conditions ranging from autoimmune to tumor settings. 


\section{Results}

Suppressive Treg from human inflamed joint fluid demonstrate a distinct transcriptional profile with an enhanced core Treg signature

To determine whether the gene expression profile of human Treg in an inflammatory environment is different from circulating human Treg, $\mathrm{CD} 3^{+} \mathrm{CD} 4^{+} \mathrm{CD} 25^{+} \mathrm{CD} 127^{\text {low }}$ Treg and $\mathrm{CD}^{+} \mathrm{CD} 4^{+} \mathrm{CD} 25^{-} \mathrm{CD} 127^{+}$non-Treg were isolated from: synovial exudate obtained from inflamed joints of JIA patients, peripheral blood (PB) from JIA patients with active and inactive disease, and PB from healthy children and healthy adults (Supplementary Fig. 1a for gating strategy). More than $90 \%$ of the sorted Treg populations were FOXP3 positive (Supplementary Fig. 1b). The transcriptional landscape was determined with RNA-sequencing. An unsupervised principal component analysis (PCA) was performed to study the variability between Treg derived from different environments. SF-derived Treg clearly clustered separately from PBderived Treg (Fig. 1a), indicating that SF-derived Treg have a specific expression pattern compared to PB-derived Treg.

In accordance with previous publications we confirmed that SF-derived Treg were functional using an in vitro suppression assay (Fig. 1b) ${ }^{16,17}$. As expected, Treg signature genes were significantly enriched in SF Treg compared to SF $\mathrm{CD}^{+}$non-Treg (Fig. 1c, left panel). Importantly, Treg signature genes were also enriched in SF compared to PB Treg derived from both healthy children and JIA patients (Fig. 1c right panel and Supplementary Fig. 2a). These included Treg hallmark genes important for Treg stability and function such as FOXP3, CTLA4 and TIGIT, at both the transcriptional and protein level (Fig. 1d, e and Supplementary Fig. 1a and 2b) ${ }^{18,19}$. In humans, $\mathrm{CD}^{+} \mathrm{CD}^{+}$non-Treg can upregulate FOXP3 and associated markers that as 
such can serve as markers for $\mathrm{T}$ cell activation ${ }^{3}$. We indeed observed a slight upregulation of FOXP3, CTLA4 and TIGIT at mRNA and protein level in SF non-Treg but not near the levels observed in SF Treg (Fig. 1d, e and Supplementary Fig. 1b and 2b), further confirming that SF Treg and non-Treg are distinct cell populations. Together these data demonstrate that the inflammatory environment reinforces the Treg-associated program.

Inflammatory environment derived Treg display a specific effector profile

A pairwise comparison between SF- and PB-derived Treg from healthy children revealed many differentially expressed genes, including core Treg markers including FOXP3 and CTLA4, but also markers that reflect more differentiated Treg, like PRDM1 (encoding Blimp-1), ICOS, $B A T F$ and $B A C H 2$ (Fig. 2a, Supplementary Table 1). Based on recent literature we analyzed the expression of markers related to eTreg cell differentiation in mice. Hierarchical clustering analysis confirmed that Treg clustered separately from non-Treg and revealed a cluster of core Treg genes with increased expression in all Treg groups including CTLA4, FOXP3, IL2RA (encoding CD25), TIGIT and IKZF4 (encoding Helios) (Fig. 2b, box 1). Furthermore, clustering revealed genes that show molecular heterogeneity within the Treg groups. Some markers were expressed almost exclusively by SF Treg (including GMZB, ICOS and IL10) whereas others were highly expressed by SF Treg but with shared, albeit lower expression by SF non-Treg (including PRDM1, BATF, TNFRSF18 encoding GITR, TNFRSF1B and HAVCR2 encoding TIM-3) (Fig. 2b, box 2). A third cluster concerns genes most highly expressed in non-Treg (Fig. 2b, box 3). Some genes in this cluster shared expression with SF Treg, such as LAG3, CXCR3 and PDCD1 (encoding PD-1) whereas expression of CD4OLG, BACH2, SATB1, CCR7 and TCF7 was clearly lower in SF Treg (Fig. 2b, box 2 and 3). Interestingly, the more 
heterogeneously expressed genes listed in box 2 and 3 were previously associated with an eTreg profile, including ICOS, IL10, PRDM1, TNRFSF18 and PDCD1. The increased expression of ICOS, GITR and PD-1 was verified at protein level (Fig. 2c and Supplementary Fig. 3a). Besides the differential expression of these markers, we found a significant enrichment of genes within SF Treg recently described in mice to be up- or downregulated in $\mathrm{eTreg}^{20}$ (Fig. 2d and Supplementary Fig. 3b), confirming the eTreg profile. Collectively, these data demonstrate that autoimmune-inflammation derived human Treg display an eTreg signature.

\section{SF Treg demonstrate adaptation to the interferon-skewed inflammatory environment}

To determine the relationship between inflammatory environment-derived and PB-derived cells, we performed unsupervised PCA analysis on SF Treg and non-Treg, and PB Treg from healthy children. PB Treg were clearly separated from SF-derived cells, confirming that environment plays a dominant role in determining the transcriptional landscape (Fig. 3a). K-mean clustering analysis showed that SF Treg and non-Treg share increased expression of genes related to inflammation-associated pathways, including pathways linked to cytokine responses such as Interferon and IL-12 (Supplementary Fig. 4a). K-mean, and subsequent gene ontology analysis of SF Treg and all PB Treg groups derived from children demonstrated that pathways associated with Th1-skewing were specifically upregulated in SF Treg (Fig. 3b and Supplementary Fig. 4b, c). Indeed, expression of the Th1 key transcription factor TBX21 (T-bet), the Th1 related chemokine receptor $C X C R 3$, and IL-12 receptor $\beta 2$ (IL12RB2) in SF Treg was increased on both mRNA and protein level (Fig. 3c, d and Supplementary Fig. 4d). Expression of $T B X 21$ and $C X C R 3$ was equally high in both SF Treg and non-Treg, whilst IL12RB2 showed a significantly higher expression in SF Treg (adjusted p-value $=6.8 \mathrm{E}^{-10}$ ). Accordingly, SF Treg in contrast to 
non-Treg showed co-expression of T-bet and FOXP3 protein excluding significant contamination of non-Treg potentially contributing to the high T-bet levels observed in SF Treg (Fig. 3e). These results indicate that SF Treg exhibit functional specialization to allow regulation of specific Th cell responses at particular tissue sites, as was previously established in mice ${ }^{21-23}$. In line herewith, we found an enrichment in SF Treg of the transcriptional signature of $\mathrm{TIGIT}^{+}$ Treg which have been recently identified as activated Treg selectively suppressing Th1 and Th17 cells in mice ${ }^{19}$ (Supplementary Fig. 4e).

The high expression of Th1-related proteins raised the question whether SF Treg may have acquired a Th1 phenotype. However, SF Treg failed to produce both IL-2 and IFN $\gamma$ (Fig. 3f and Supplementary Fig. 4f) and responded dose-dependently to IL-2 with increasing pSTAT5 levels (Supplementary Fig. 4g), a signaling pathway pivotal for Treg survival and function ${ }^{24}$. In fact, compared to PB Treg, SF Treg appeared to be even more responsive to IL-2. Altogether, our findings demonstrate adaptation of SF Treg to their inflammatory environment while maintaining Treg key features.

Effector Treg differentiation and inflammation are regulated by the (super-)enhancer landscape

To explore the mechanistic regulation of the inflammation-adapted eTreg profile we analyzed the enhancer landscape of SF Treg. Enhancers are distal regulatory elements in the DNA that allow binding of transcription factors and as such coordinate gene expression. Epigenetic regulation of enhancers is critical for context-specific gene regulation ${ }^{25}$. Enhancers can be defined by areas enriched for monomethylation of lysine 4 on histone H3 (H3K4me1 enrichment) and acetylation of lysine 27 on histone H3 (H3K27ac enrichment), where H3K27Ac identifies active enhancers $^{26}$. ChIP-seq performed for H3K27ac and H3K4me1 using SF Treg and healthy adult 
PB Treg showed a striking difference in enhancer profile and activity, with 3333 out of 37307 and 970 out of 10991 different peaks called between SF and PB Treg for H3K27ac and H3K4me1, respectively (Fig. 4a, Supplementary Fig. 5b, upper panel and Supplementary Table 2). We next assessed if the transcriptome of SF Treg is reflected at an epigenetic level. Genes that demonstrated increased $\mathrm{H} 3 \mathrm{~K} 27 \mathrm{ac}$ and/or H3K4me1 were increased at the mRNA level and vice versa (Fig. 4a, Supplementary Fig. 5b, middle and lowest panel and Supplementary Table 2), confirming that gene expression and chromatin-acetylation and -monomethylation are interconnected in these cells.

Super-enhancers are large clusters of enhancers that specifically regulate genes defining cell identity, both in health and disease ${ }^{27,28}$. Also at super-enhancer level, we found significant enrichment in the transcribed genes, again indicating that the SF Treg profile is mediated by epigenetic changes (Fig. 4b and Supplementary Fig. 5c, middle and lowest panel). The analysis of differential super-enhancer-associated genes revealed 337 out of 791 and 317 out of 713 different gene loci for H3K27ac and H3K4me1, respectively (Fig. 4b and Supplementary Fig. 5c, upper panel). Specifically, we identified that super-enhancers associated with genes related to canonical Th1 differentiation, such as $T B X 21$ and IL12RB2, are increased in SF Treg (Fig. 4c) demonstrating epigenetic regulation of the environment-associated profile. Core Treg genes encoding the effector molecules ICOS, IL10, CTLA4, TNFRSF18 and PDCD1 were associated with increased super-enhancers as well as FURIN and ID2 which are more putative functional Treg markers. Importantly, eTreg differentiation was also reflected in the enhancer profile of SF Treg, with differential expression of super-enhancer-associated transcriptional regulators including $B A T F, B A C H 2, S A T B 1, T R A F 3$ and SOCS1. Moreover, we found super-enhancers associated with markers not previously related to (e)Treg differentiation and mostly specific for 
human Treg, including $V D R, R X R A$ (encoding Retinoic acid receptor RXR-alpha), KAT2B (encoding p300/CBP-associated factor (PCAF)), TOX2 (encoding TOX High Mobility Group Box Family Member 2) and IL12RB2. Inflammation related homing markers (CCR2, CCR5, CCR7) were associated with differential H3K27ac levels in SF Treg as well. Finally, regulation of cell cycling and apoptosis was also mirrored in the super-enhancer landscape of SF compared to PB Treg by increased activity of DPH5, MICAL2, PHLDA1 and CCND2. Altogether, these super-enhancer-associated genes reflect adaptation and specialization of Treg within an inflammatory environment.

Motif analysis for in silico prediction of transcription factor binding sites in (super-)enhancers specifically upregulated in SF compared to PB Treg revealed motifs for Treg-specific transcription factors. These included STAT5 and Myb; the latter recently described as a core transcription factor in eTreg differentiation ${ }^{29}$. Recent papers further demonstrated BATF and RelA as crucial regulators for eTreg in mice ${ }^{30,31}$. In support hereof, we found significant enrichment of BATF, TF65 (encoding RelA), and VDR motifs within the (super-)enhancer regions specifically upregulated in SF Treg (Fig. 4d and Supplementary Fig. 5d), thereby further strengthening the eTreg profile of SF Treg. The most prevalent motifs belong to the activator protein 1 (AP-1) transcription factor subfamily, part of the basic leucine zipper (bZIP) family (Supplementary Fig. 5d) indicating this subfamily is crucial in driving the eTreg signature, as similarly proposed by DiSpirito et $a .^{32}$ for pan-tissue Treg. Moreover, within the superenhancers of both the transcriptional regulators VDR and BATF the binding sites for each of them were found, indicating that BATF (in complex with other proteins) and VDR regulate their own gene expression, and also control other eTreg genes (Fig. 4e). Together, these observations 
demonstrate that the inflammation-adapted effector phenotype of SF Treg is regulated at an epigenetic level, with a super-enhancer profile and enrichment of binding sites for eTreg genes.

The vitamin D3 receptor is a predicted key regulator of effector Treg differentiation

To extract key gene regulators in the differentiation from healthy PB to SF Treg a data-driven network and enrichment approach was performed (RegEnrich). The top predicted regulators driving the differentiation from PB to SF Treg were found to be TCF7, LEF1, JUN, SPEN (negative) and ENO1, THRAP3 and VDR (positive) (Fig. 5a, b). SPEN, THRAP3 and VDR have not been previously associated with eTreg differentiation. $V D R$ associated downstream with core Treg genes including FOXP3 and STAT3, but also Tbx21 (Fig. 5a, c and Supplementary Table 3). In line herewith, there was a strong positive correlation for both FOXP3 $(r=0.772, p<0.0001)$ and T-bet $(r=0.937, \mathrm{p}<0.0001)$ with VDR protein expression (Fig. 5e) reinforcing the predicted regulator network.

The effector Treg profile overlaps with other chronic inflammatory diseases and the human tumor Treg signature

To investigate whether the program identified in SF Treg is universal for human Treg exposed to inflammation, we compared our findings with Treg derived from PB and inflammatory joints of RA patients. Indeed, also in Treg from inflammatory exudate of RA patients similar eTreg genes were upregulated (Fig. 6a).

We then compared our data to recently published human tumor-infiltrating Treg specific signatures $^{13-15}$. Strikingly, the tumor-infiltrating Treg signature from De Simone et al. was enriched in SF Treg (Normalized Enrichment Score (NES) 1.27, FDR statistical value (FDRq) 
0.071; Fig. 6b). Vice versa, the differentially expressed genes in SF Treg versus PB Treg were enriched in tumor-infiltrating compared to normal tissue Treg (Plitas et al., 2016: NES 1.69, FDRq 0.017, Magnuson et al., 2018: NES 1.88, FDRq 0.002; Supplementary Fig. 6a, b). Hierarchical clustering analysis of the tumor-infiltrating gene signature from De Simone et al. further revealed separate clustering of SF Treg (Fig. 6c), indicating that the high expression of the signature genes is specific for human Treg at a site that is characterized with infiltration of immune cells. We also observed a small set of genes that were not upregulated in SF Treg, e.g. CX3CR1, IL17REL, IL1RL1 and IL1RL2, implying environment-restricted adaptation (Fig. 6c). The three genes that were described as the most enriched and distinctive genes in tumorinfiltrating Treg, $L A Y N, M A G E H 1$, and $C C R 8$, were selectively and highly upregulated in SF Treg (Fig. 6d). These data demonstrate that the Treg profile we observed is not restricted to the SF exudate from JIA and RA patients. In fact, it likely represents a more global profile of human Treg in inflammatory settings, likely fine-tuned by environment specific adaptations.

To investigate which cues might be specific for SF we explored the differences between upregulated genes in SF and tumor-infiltrating Treg. Relatively few pronounced SF-specific genes were found. On gene level, cytotoxic markers including GZMM, GZMA and GNLY, related to effector cell cytolysis and self-induced apoptosis of Treg $^{33}$ were SF-specific (Supplementary Fig. 6c). Gene ontology biological process analysis further revealed that in SF both active cell cycling and apoptotic pathways were upregulated, whereas in the tumor microenvironment cell activation and effector responses were prominent (Supplementary Fig. 6d). Additionally, tumorinfiltrating Treg showed distinct expression of chemokines and chemokine receptors, e.g. IL-8 (CXCL8), CX3CR1, CXCR7 and CCR10 (Supplementary Fig. 6c), some of which have been proposed as prognostic marker and/or therapeutic target in cancer ${ }^{34}$. 
From the JIA, RA and tumor datasets we could deduce a set of genes that appear universally upregulated in eTreg, and also being reflected in the (super-)enhancer landscape of SF Treg (Fig. 6e). ICOS, BATF, MAF, TNFRSF18 and SRGN were revealed as core markers previously also related to mouse eTreg. Additionally, $I L 12 R B 2, V D R$, and $K A T 2 B$, were identified as core genes, as well as $P F K F B 3$ and $L D H A$ involved in glycolysis and the apoptosis-related genes MICAL2 and TOX2. The latter two have so far not been linked to Treg in human or mice, although both have independently been linked to human cancers ${ }^{35,36}$. Collectively, our results demonstrate that human Treg in different inflammatory settings share an effector profile and strongly indicates that inflammation drives eTreg differentiation in a comparable manner in different environments. 


\section{Discussion}

The discovery of eTreg in non-lymphoid tissues and inflammatory sites in mice have raised questions how human eTreg are transcriptionally and epigenetically programmed in different settings. The present study provides evidence that human Treg in a local inflammatory setting undergo further differentiation into specialized eTreg, with a unique expression profile. This is reflected by high expression of TBX21 and CXCR3 and core Treg markers including FOXP3, CTLA4 and TIGIT. Essential Treg features are maintained, such as suppressive function, IL-2 responsiveness and absence of cytokine production. The transcriptional changes were mirrored at an epigenetic level, both in the enhancer and super-enhancer landscape, demonstrating that the profile is highly regulated. Additionally, we identify previously unappreciated and specific markers in human eTreg, like VDR, IL12R $\beta 2$, and BATF; with VDR as a predicted keyregulator in human eTreg differentiation. Finally, we demonstrate that the profile is not limited to autoimmune inflammation in JIA and RA, but shares almost complete overlap with tumorinfiltrating Treg. The similarity between a tumor and an autoimmune setting might seem counterintuitive, since the former reflects an immune-suppressive environment whereas the latter is associated with immune activation. Both environments however share features including immune cell infiltration and inflammation ${ }^{37}$. This overlapping profile might therefore represent a more universal profile of human Treg in inflammatory environments. Because of the extensive genome-wide changes of Treg from an inflammatory environment insight in their functionality is important. In line with previous reports ${ }^{16,17}$, we show that SF Treg are indeed suppressive and 
additionally highly responsive to IL-2 by phosphorylation of STAT5, excluding impaired IL-2 signaling $^{38,39}$. This advocates against an intrinsic defect of Treg in inflammation. Maintenance of inflammation may be explained by resistance of local effector cells, previously demonstrated in both JIA and RA ${ }^{17,40,41}$.

In mice, functional specialization of Treg towards Th1 inflammation by upregulation of T-bet is described as essential to prevent Th1 autoimmunity ${ }^{9,10}$. The remarkable co-expression of T-bet and FOXP3 in SF Treg in our study, with T-bet levels as high as in SF non-Treg, implies that functional specialization is translatable to a human setting. The increased expression of migration markers such as CXCR3 and CCR5 on protein, transcriptional and enhancer level further strengthens this, as high T-bet expression is crucial for migration and thus co-localization of Th1-specific Treg and Th1 effector T cells via upregulation of these chemokine receptors ${ }^{9,10}$. Contamination of Treg with non-Treg can be a concern when sorting ex vivo human cells. However, we exclude substantial contamination because 1) our data show robust transcriptomic differences between SF Treg and non-Treg, with increased expression of Treg hallmark genes in SF Treg, 2) sorted Tregs are $>90 \% \mathrm{FOXP}^{+}, 3$ ) there is clear co-expression of FOXP3 and T-bet on the single cell level, 4) we show that SF Treg, while expressing high T-bet levels, lack cytokine production, potentially caused by high expression of FOXP3, SOCS1, EGR2 and EGR3 (Supplementary Table 1$)^{42,43}$. Nevertheless, as recently shown by Miragaia et al. $^{4}$ there may be heterogeneity within the eTreg population.

Our study is the first to investigate both the transcriptional and epigenetic regulation of eTreg in human inflammatory settings allowing us to discern human-specific eTreg regulation as well as commonalities between mice and men. The epigenetic landscape of human Treg is little explored, with only two papers studying H3K27acetylation and H3K4monomethylation of 
circulating human Treg ${ }^{44,45}$. Arvey et al. conclude that the majority of Treg lineage-specific elements are not conserved between mice and human further emphasizing the lack of knowledge of the human Treg epigenetic landscape. Gao et al. focused on SNPs within enhancers of PB Treg from Type 1 diabetes patients, and in line with our data showed, that cell cycle and apoptosis regulation is highly reflected in the enhancer landscape of these patients. These data further underscores the need for additional studies, especially in non-lymphoid tissues during non-homeostatic conditions. We found similarities between mice and men in eTreg differentiation regarding downregulation of $S A T B 1, B A C H 2, T C F 7$ and $L E F 1$, all associated with a super-enhancer in PB but not SF Treg. Although all four genes are crucial for Treg development, recent reports show that downregulation of these regulators is necessary for further differentiation of Treg and preventing conversion into Th cells ${ }^{46-52}$. BACH2 can transcriptionally repress PRDM1 (encoding Blimp-1) in $\mathrm{T}$ cells ${ }^{50}$, and in line herewith we observed increased acetylation and gene expression of PRDM1 in SF Treg. This was further supported by the decrease in $T C F 7$ and $L E F 1$, both negatively correlating with eTreg marker expression including $P R D M 1^{52}$. Moreover, we found BATF as a prominent marker in SF Treg, with increased expression on both transcriptional and epigenetic level, and a binding site in upregulated superenhancer associated genes. In addition, high BATF expression was also observed in RA SF Treg and tumor-infiltrating Treg, suggesting BATF is a key transcriptional regulator in both human and mice eTreg. We also found remarkable differences in eTreg signature markers compared to what is known from mice. Most pronounced is upregulation of IL12RB2 at both the transcriptional and epigenetic level, and a binding site in upregulated super-enhancer associated genes, whereas in mice eTreg this marker is downregulated. Instead, impaired IL-12R 32 expression has been reported as a key checkpoint preventing Treg from fully differentiating 
towards Th1 cells in mice ${ }^{23}$. However, our data are in line with recent reports showing selective expression of IL12RB2 in human PB Treg ${ }^{44,53}$, suggesting this marker has different functions in human and mice.

Another unexpected finding is the upregulation of VDR in both JIA and RA SF Treg, on both transcriptional and epigenetic level, as well as in tumor-infiltrating Treg. Although not highlighted, high VDR levels were present in breast tumor-infiltrating Treg $^{14}$ and uterine eTreg ${ }^{54}$. Besides the well-known tolerogenic effects of vitamin $\mathrm{D}_{3}{ }^{55}$, VDR is not well-studied in the Treg context, especially in humans. A recent study did show that memory CCR6 ${ }^{+} \mathrm{Th}$ cells gained a suppressive phenotype, including CTLA-4 expression, and functional suppressive capacity similar to Treg upon incubation with vitamin $\mathrm{D}_{3}{ }^{56}$. Here we show VDR is a predicted key-regulator in SF eTreg, positively correlated with both FOXP3 and T-bet expression.

Another striking difference is absence of KLRG1 upregulation in human eTreg. In mice, KLRG1 is a key marker to identify eTreg, although not crucial for eTreg function ${ }^{57}$. KLRG1 is not upregulated on SF Treg and associated with a super-enhancer only in PB Treg. Additionally, in our data gene expression of KLRG1 is restricted to non-Treg (both SF and PB). Confusing the findings are discrepancies possibly caused by the tissue measured or comparison made. Miragaia et $a l .^{4}$ showed upregulation of PIM1 in non-lymphoid Treg compared to PB Treg in mice, but PIM1 was not expressed in human tissue Treg. In our data however, PIM1 is highly increased in SF compared to PB Treg of healthy adults and children (Supplementary Table 1). In summary, we show that human eTreg show similarities in eTreg differentiation markers identified in mice, but also found human-specific eTreg markers including IL-12R $\beta 2$ and VDR, and absence of KLRG1. 
Previous research has shown that ex vivo human Treg are highly glycolytic ${ }^{58}$, in line with their high proliferative capacity ${ }^{59}$. In mice increased glycolysis is required for eTreg differentiation and migration to inflammatory sites ${ }^{60,61}$. In support hereof, glycolysis-associated genes including PFKFB3, LDHA, and PKM2 were increased in SF Treg on both RNA and (super-)enhancer level. Additionally, preliminary analysis of T-cell glucose consumption by Seahorse technology showed increased glycolysis in SF compared to PB Treg (data not shown). Finally, ENO1, encoding the glycolytic enzyme Enolase 1, is a predicted key-regulator of eTreg differentiation. Lately, application of Treg-based therapies for autoimmune diseases and transplantation settings is gaining renewed interest. Promising data from animal models and clinical trials, both with cell therapy involving adoptive transfer and with low dose IL-2 administration, pave the way for Treg therapies to reach the clinic $^{62}$. Our data show that circulating human Treg are markedly different from their counterparts derived from sites characterized by immune activation. This is reflected in the expression of effector markers and transcriptional regulators, but also in the expression of specific chemokine receptors. Regarding Treg-based therapies appreciating that specific environments may require adapted Treg, both for migration and function, is important. Moreover, in this study we extensively profiled human eTreg abundantly present at sites of inflammation. This information can form a basis for follow-up studies that may eventually allow characterization of small amounts of circulating eTreg, for example to monitor patients undergoing treatment.

In conclusion, our study is the first to uncover the transcriptional and epigenetic program that defines human inflammatory Treg. SF Treg display an environment-adapted as well as an eTreg phenotype established at the epigenetic level. Moreover, we describe striking similarities of the eTreg program with tumor-infiltrating Treg and SF Treg from RA patients. This revealed a set of 
genes shared with human eTreg from affected sites in JIA, RA and cancer including BATF, VDR, MICAL2, TOX2, KAT2B, PFKFB3 and IL12Rß2. Finally, we show that THRAP3, ENO1 and VDR are predicted key-regulators driving human SF eTreg differentiation at the transcriptomic level.

\section{Methods}

\section{Collection of SF and PB Samples}

Patients with JIA ( $n=41)$ were enrolled by the Pediatric Rheumatology Department at University Medical Center of Utrecht (The Netherlands). Of the JIA patients $n=8$ were diagnosed with extended oligo JIA and $n=33$ with oligo JIA, according to the revised criteria for JIA ${ }^{63}$, with an average age of 11.3 years (range 3.2-19 years) and a disease duration at the time of inclusion of 4.9 years (range $0.1-15$ years). Patients with RA $(n=7)$ were enrolled by the rheumatology outpatient clinic at Guy's and St. Thomas' Hospital NHS Trust (United Kingdom). The average age of RA patients was 61 years (range 30-75 years). PB and synovial fluid (SF) was obtained when patients visit the outpatient clinic via vein puncture or intravenous drip, and by therapeutic joint aspiration of the affected joints, respectively. The study was conducted in accordance with the Institutional Review Board of the University Medical Center Utrecht (approval no. 11-499/C; JIA) and the Bromley Research Ethics Committee (approval no. 06/Q0705/20; RA). PB from healthy adult volunteers ( $\mathrm{HC}, \mathrm{n}=20$, average age 41.7 years with range 27-62 years) was obtained from the Mini Donor Service at University Medical Center Utrecht. PB from $n=8$ healthy children (average age 11.4 years with range 7.3-15.6 years) was obtained from a cohort of control subjects for a case-control clinical study. 


\section{Isolation of SFMC and PBMC}

SF of JIA patients was incubated with hyaluronidase (Sigma-Aldrich) for $30 \mathrm{~min}$ at $37^{\circ} \mathrm{C}$ to break down hyaluronic acid. Synovial fluid mononuclear cells (SFMCs) and peripheral blood mononuclear cells (PBMCs) were isolated using Ficoll Isopaque density gradient centrifugation (GE Healthcare Bio-Sciences, AB) and were used after freezing in Fetal Calf Serum (FCS) (Invitrogen) containing 10\% DMSO (Sigma-Aldrich).

\section{Suppression assay}

$\mathrm{CD}^{+} \mathrm{CD} 4^{+} \mathrm{CD} 25^{+} \mathrm{CD} 127^{\text {low }}$ cells (Treg) were isolated from frozen PBMC, using the FACS Aria III (BD). Antibodies used for sorting are: anti-human CD3-BV510 (OKT3), CD25-PE/Cy7 (MA251; BD), CD127-AF647 (HCD127; Biolegend), CD4-FITC (RPA-T4; eBioscience). To check for FOXP3 expression of the sorted populations cells were fixed and permeabilized by using eBioscience Fixation and Permeabilization buffers (Invitrogen) and stained with anti-human FOXP3-eF450 (PCH101; eBioscience). Read out of proliferation is performed with the following antibodies: CD3-PerCP/Cy5.5 (UCHT1; Biolegend), CD4-FITC (RPA-T4; eBioscience), CD8APC (SK1; BD). Total PBMC were labeled with $2 \mu \mathrm{M}$ ctViolet (Thermo Fisher) and cultured alone or with different ratios of sorted Treg $(1: 16,1: 8,1: 4,1: 2)$. Cells were cultured in RPMI1640 media containing 10\% human AB serum with addition of L-Glutamine and Penicillin/Streptomycin. PBMC were stimulated by $0,1 \mu \mathrm{g} / \mathrm{ml}$ coated anti-CD3 (eBioscience) and incubated for four days in a 96 well round bottom plate (Nunc) at $37^{\circ} \mathrm{C}$. After 4 days cells were stained with CD3, CD4, and CD8 for read out of proliferation by flow cytometry performed 
on FACS Canto II (BD Biosciences) and data was analyzed using FlowJo Software (Tree Star Inc.).

\section{STAT5 Phosflow}

PBMC and SFMC were thawed and resuspended in PBS $\left(0,5-1,0 \times 10^{6}\right.$ living cells/tube). Surface staining of CD3 (Biolegend) and CD4 (eBioscience) was performed for 25 min at $4{ }^{\circ} \mathrm{C}$. Cells were then stimulated with $0,1.0,10$ or $100 \mathrm{IU} / \mathrm{ml}$ human (h)IL-2 (Proleukin; Novartis) for $30 \mathrm{~min}$ at $37^{\circ} \mathrm{C}$, fixated and permeabilized by using buffers from the Transcription Factor Phospho Buffer Set (BD Biosciences). Intranuclear staining of FOXP3-eF450 (PCH101), T-beteF660 (eBio4B10; eBioscience), CD25-PE/CY7 (M-A251) and pSTAT5-PE (pY695; BD) was performed for $50 \mathrm{~min}$ at $4^{\circ} \mathrm{C}$. Data acquisition and analysis as above.

\section{RNA-sequencing}

$\mathrm{CD} 3^{+} \mathrm{CD} 4^{+} \mathrm{CD} 25^{+} \mathrm{CD} 127^{\text {low }}$ and $\mathrm{CD} 3^{+} \mathrm{CD} 4^{+} \mathrm{CD} 25^{-} \mathrm{CD} 127^{+}$cells were sorted by flow cytometry from HC PBMC and JIA patient SFMC and PBMC. Total RNA was extracted using the AllPrep DNA/RNA/miRNA Universal Kit (Qiagen) as specified by the manufacturer's instructions and stored at $-80^{\circ} \mathrm{C}$. Sequencing libraries were prepared using the Rapid Directional RNA-Seq Kit (NEXTflex). Libraries were sequenced using the Nextseq500 platform (Illumina), producing single end reads of $75 \mathrm{bp}$ (Utrecht Sequencing Facility). Sequencing reads were mapped against the reference human genome (hg19, NCBI37) using BWA (v0.7.5a, mem -t 7 -c $100-\mathrm{M}-\mathrm{R}$ ) (see Supplementary table 4 for quality control information). Differential gene expression was performed using DEseq2 and custom Perl and $\mathrm{R}$ (www.r-project.org) scripts (https://github.com/UMCUGenetics/RNASeq). For K-means clustering and PCA analysis, genes 
with fold change between samples on 10th and 90th quantile at least $1 \log 2$ RPKM and expression at least $2 \log 2 \mathrm{RPKM}$ in the sample with the maximal expression were used. K-means clustering was done on gene expression medians per group, with an empirically chosen k of 14 . Gene Ontology pathway analyses were performed using ToppFun (https://toppgene.cchmc.org/enrichment.jsp) with as input genes belonging to the defined k-mean clusters, with an FDR-corrected p-value $<0.05$ defining significance. Gene set enrichment analysis (GSEA) $)^{64}$, with as input the $\log 2$ RPKM data, was used to assess whether specific signatures were significantly enriched in one of the subsets. One thousand random permutations of the phenotypic subgroups were used to establish a null distribution of enrichment score against which a normalized enrichment score and FDR-corrected q values were calculated. Gene-sets were either obtained by analyzing raw data using GEO2R (NCBI tool) or downloaded from published papers, or self-made based on the $\mathrm{H} 3 \mathrm{~K} 27 \mathrm{ac} / \mathrm{H} 3 \mathrm{~K} 4 \mathrm{me} 1$ data. In particular, the following published data sets were used: human core Treg signature: Ferraro et al. ${ }^{65}$; effector Treg signature in mice: Dias et al. ${ }^{29}$; tumor-infiltrating Treg signature: De Simone et al. ${ }^{13}$, Plitas et $a l .{ }^{14}$, Magnuson et al. ${ }^{15}$; effector Treg genes in mice: gene set GEO: GSE61077; TIGIT $^{+}$Treg signature in mice: Joller et al. ${ }^{19}$. Identification of key-regulators was performed using RegEnrich (https://bitbucket.org/systemsimmunology/regenrich/src/master/) based on the differential gene expression data followed by unsupervised WGCNA for the network inference, mean RPKM counts $>0$ were included, and Fisher's exact test was used for enrichment analysis. Heatmaps and subsequent hierarchical clustering analyses using One minus Pearson correlation were performed using Morpheus software (https://software.broadinstitute.org/morpheus/).

\section{H3K27ac and H3K4me1 ChIP-sequencing}


ChIP-seq was performed as described previously by Peeters et al. ${ }^{66}$. In short, PBMC from HC and SFMC from JIA patients were thawed and $0.5-1$ million $\mathrm{CD} 3^{+} \mathrm{CD} 4^{+} \mathrm{CD} 25^{+} \mathrm{CD} 127^{\text {low }}$ cells were sorted by flow cytometry. For each sample, cells were crosslinked with $2 \%$ formaldehyde and crosslinking was stopped by adding $0.2 \mathrm{M}$ glycine. Nuclei were isolated in $50 \mathrm{mM}$ Tris $(\mathrm{pH}$ 7.5), $150 \mathrm{mM} \mathrm{NaCl}, 5 \mathrm{mM}$ EDTA, $0.5 \%$ NP-40, and 1\% Triton X-100 and lysed in $20 \mathrm{mM}$ Tris (pH 7.5), 150mMNaCl, 2mMEDTA, 1\% NP-40, 0.3\% SDS. Lysates were sheared using Covaris microTUBE (duty cycle 20\%, intensity 3, 200 cycles per burst, 60-s cycle time, eight cycles) and diluted in $20 \mathrm{mM}$ Tris (pH 8.0), $150 \mathrm{mM} \mathrm{NaCl}, 2 \mathrm{mM}$ EDTA, 1\% X-100. Sheared DNA was incubated overnight with anti-histone H3 acetyl K27 antibody (ab4729; Abcam) or anti-histone H3 (mono methyl K4) antibody (ab8895; Abcam) pre-coupled to protein A/G magnetic beads. Cells were washed and crosslinking was reversed by adding $1 \%$ SDS, 100mM NaHCO3, $200 \mathrm{mM} \mathrm{NaCl}$, and $300 \mu \mathrm{g} / \mathrm{ml}$ proteinase $\mathrm{K}$. DNA was purified using ChIP DNA Clean \& Concentrator kit (Zymo Research), end-repair, a-tailing, and ligation of sequence adaptors was done using Truseq nano DNA sample preparation kit (Illumina). Samples were PCR amplified, checked for the proper size range and for the absence of adaptor dimers on a $2 \%$ agarose gel and barcoded libraries were sequenced 75 bp single-end on Illumina NextSeq500 sequencer (Utrecht DNA sequencing facility).

Sample demultiplexing and read quality assessment was performed using BaseSpace (Illumina) software. Reads with quality score of Q>30 were used for downstream analysis. Reads were mapped to the reference genome (hg19/hg38) with Bowtie 2.1.0 using default settings for H3K27ac ChIP-seq and BWA for H3K4me1 ChIP-seq (see Supplementary Table 4 for quality control information). SAM files were converted to BAM files using samtools version 0.1.19. Peaks were subsequently called using MACS-2.1.0. Enriched regions were identified compared 
to the input control using MACS2 callpeak--nomodel --exttsize 300 --gsize=hs -p 1e-9. The mapped reads were extended by $300 \mathrm{bp}$ and converted to TDF files with igvtools-2.3.36 and were visualized with IGV-2.7.2 $2^{67}$ for H3K27ac ChIP-seq and H3K4me1 ChIP-seq. Differential binding analysis was performed using the $\mathrm{R}$ package DiffBind v1.8.5. In DiffBind read normalization was performed using the TMM technique using reads mapped to peaks which were background subtracted using the input control. Enhancer gene associations were determined as the nearest TSS to the center of the enhancer and super-enhancer locus. Super-enhancers were identified by employing the ROSE algorithm ${ }^{68}$ using a stitching distance of the MACS2 called peaks of $12.5 \mathrm{~kb}$, peaks were excluded that were fully contained in the region spanning 1000bp upstream and downstream of an annotated TSS (-t 1000). The H3K27ac/H3K4me1 signal was corrected for background using the input control and subsequently ranked by increasing signal (Fig. S5A). Super-enhancer gene associations were determined as the nearest TSS to the center of the enhancer and super-enhancer locus. BEDtools v2.17.0 was used for general manipulation of peak bed-files. Motif enrichment analysis was performed using the HOMER software v4.11 (findMotifsGenome.pl; hg19/hg38; -size 200). ChIP-seq data for activated Treg, VDR and BATF was retrieved from GEO:GSE43119, GSE89431 and GSE32465, respectively.

\section{Vitamin D receptor and 1,25 vitamin D3 incubation assay}

Thawed HC PBMC and JIA patient SFMC were stained by surface and intranuclear staining as described above with the following antibodies: anti-human fixable viability dye eF506, FOXP3PerCP/Cy5.5 (PCH101; eBioscience), CD3-AF700 (UCHT1), CD4-BV785 (OKT4; Biolegend), CD25-BV711 (2A3), CD127-BV605 (A019D5; Sony Biotechnology), and VDR-PE (D-6; Santa Cruz Biotechnology). Data acquisition and analysis as above. 


\section{Microarray RA Treg}

$\mathrm{CD}_{14}{ }^{+}$monocytes (purity $>98 \%$ ) were depleted through positive selection using CD14 MicroBeads (Miltenyi Biotec). $\mathrm{CD}^{+} \mathrm{T}$ cells were isolated from the $\mathrm{CD} 14^{-}$cell fraction by negative selection (Miltenyi Biotec) and stained with CD4-PerCP/Cy5.5 (SK3), CD45RAAPC/Cy7 (HI100), CD45RO-PB (UCHL1), CD127-FITC (A019D5; BioLegend), and CD25-PE (REA945; Miltenyi Biotec). $\mathrm{CD}^{+} \mathrm{CD} 45 \mathrm{RA}^{-} \mathrm{CD} 45 \mathrm{RO}^{+} \mathrm{CD} 25^{+} \mathrm{CD} 127^{\text {low }}$ cells (memory Treg cells), were sorted using a BD FACSAria II. Sorted Treg cell samples were lysed in $1000 \mu$ of TRIzol (Invitrogen). Chloroform $(200 \mu \mathrm{l})$ was added, and the samples were then whirl mixed and incubated for 2-3 minutes at room temperature. Following centrifugation $(10,000 \mathrm{~g}$ for 15 minutes at $4^{\circ} \mathrm{C}$ ), the water phase was further purified using the ReliaPrepTM RNA Miniprep system (Promega) per manufacturer's instructions. RNA integrity was confirmed on an Agilent Technologies 2100 bioanalyzer. One hundred nanograms of total RNA was used to prepare the targets (Affymetrix) in accordance with the manufacturer's instructions. Hybridization cocktails were hybridized onto a Human Gene 2.0 ST Array. Chips were scanned and gene expression data were normalized using the RMA algorithm. Gene expression analysis was performed using Qlucore Omics Explorer software, version 3.0.

\section{Statistical analysis}

For ChIP-seq and RNA-seq analysis, p-values were adjusted with the Benjamini-Hochberg procedure. Protein and cytokine expression was analyzed with Pearson's correlation, two-tailed Mann-Whitney test, Two-way ANOVA with Sidak correction for multiple testing or a mixedeffects model with Dunnett post-hoc on paired data with missing values using GraphPad Prism. 


\section{Data availability}

The authors declare that all data supporting the findings of this study are available within the article and its Supplementary information files or from the corresponding author upon reasonable request. RNA-seq and ChIP-seq raw data is deposited in the Gene Expression Omnibus and will be made publicly available upon acceptance in a journal.

\section{References}

1. Brunkow, M. E. et al. Disruption of a new forkhead/winged-helix protein, scurfin, results in the fatal lymphoproliferative disorder of the scurfy mouse. Nat. Genet. 27, 68-73 (2001).

2. Ochs, H. D. et al. The immune dysregulation, polyendocrinopathy, enteropathy, X-linked syndrome (IPEX) is caused by mutations of FOXP3. Nat. Genet. 27, 20-21 (2001).

3. Cretney, E. et al. The transcription factors Blimp-1 and IRF4 jointly control the differentiation and function of effector regulatory T cells. Nat Immunol 12, 304-311 (2011).

4. Miragaia, R. J. et al. Single-Cell Transcriptomics of Regulatory T Cells Reveals Trajectories of Tissue Adaptation. Immunity 50, 493-504.e7 (2019).

5. Liston, A. \& Gray, D. H. D. Homeostatic control of regulatory T cell diversity. Nat. Rev. Immunol. 14, 154-165 (2014).

6. Vasanthakumar, A. et al. The transcriptional regulators IRF4, BATF and IL-33 orchestrate development and maintenance of adipose tissue-resident regulatory T cells. Nat. Immunol. 16, 276-285 (2015). 
7. Povoleri, G. A. M. et al. Human retinoic acid-regulated CD161 + regulatory T cells support wound repair in intestinal mucosa. Nat. Immunol. 19, 1403-1414 (2018).

8. Kalekar, L. A. et al. Regulatory T cells in skin are uniquely poised to suppress profibrotic immune responses. Sci. Immunol. 4, aaw2910 (2019).

9. Koch, M. A. et al. The transcription factor T-bet controls regulatory T cell homeostasis and function during type 1 inflammation. Nat. Immunol. 10, 595-602 (2009).

10. Levine, A. G. et al. Stability and function of regulatory T cells expressing the transcription factor T-bet. Nature 546, 421-425 (2017).

11. Miyara, M. et al. Functional Delineation and Differentiation Dynamics of Human CD4+ T Cells Expressing the FoxP3 Transcription Factor. Immunity 30, 899-911 (2009).

12. Panduro, M., Benoist, C. \& Mathis, D. Tissue Tregs. Annu. Rev. Immunol. 34, 609-633 (2016).

13. De Simone, M. et al. Transcriptional Landscape of Human Tissue Lymphocytes Unveils Uniqueness of Tumor-Infiltrating T Regulatory Cells. Immunity 45, 1135-1147 (2016).

14. Plitas, G. et al. Regulatory T Cells Exhibit Distinct Features in Human Breast Cancer. Immunity 45, 1122-1134 (2016).

15. Magnuson, A. M. et al. Identification and validation of a tumor-infiltrating Treg transcriptional signature conserved across species and tumor types. Proc. Natl. Acad. Sci. U. S. A. 115, E10672-E10681 (2018).

16. Van Amelsfort, J. M. R., Jacobs, K. M. G., Bijlsma, J. W. J., Lafeber, F. P. J. G. \& Taams, L. S. CD4+CD25+ regulatory T cells in rheumatoid arthritis: Differences in the presence, phenotype, and function between peripheral blood and synovial fluid. Arthritis Rheum. 50, 2775-2785 (2004). 
17. Wehrens, E. J. et al. Functional human regulatory T cells fail to control autoimmune inflammation due to PKB/c-akt hyperactivation in effector cells. Blood 118, 3538-3548 (2011).

18. Teh, P. P., Vasanthakumar, A. \& Kallies, A. Development and Function of Effector Regulatory T Cells. in Progress in Molecular Biology and Translational Science (ed. Liston, A.) 155-174 (ScienceDirect, 2015). doi:10.1016/bs.pmbts.2015.08.005.

19. Joller, N. et al. Treg cells expressing the coinhibitory molecule TIGIT selectively inhibit proinflammatory Th1 and Th17 cell responses. Immunity 40, 569-581 (2014).

20. Levine, A. G., Arvey, A., Jin, W. \& Rudensky, A. Y. Continuous requirement for the TCR in regulatory T cell function. Nat. Immunol. 15, 1070-1078 (2014).

21. Chaudhry, A. et al. CD4+ regulatory T cells control TH17 responses in a Stat3-dependent manner. Science 326, 986-991 (2009).

22. Zheng, Y. et al. Regulatory T-cell suppressor program co-opts transcription factor IRF4 to control T(H)2 responses. Nature 458, 351-356 (2009).

23. Koch, M. A. et al. T-bet+ Treg Cells Undergo Abortive Th1 Cell Differentiation due to Impaired Expression of IL-12 Receptor $\beta 2$. Immunity 37, 501-510 (2012).

24. Chinen, T. et al. An essential role for the IL-2 receptor in Treg cell function. Nat. Immunol. 17, 1322-1333 (2016).

25. Heintzman, N. D. et al. Histone modifications at human enhancers reflect global cell-typespecific gene expression. Nature 459, 108-112 (2009).

26. Creyghton, M. P. et al. Histone H3K27ac separates active from poised enhancers and predicts developmental state. Proc. Natl. Acad. Sci. U. S. A. 107, 21931-21936 (2010).

27. Hnisz, D. et al. XSuper-enhancers in the control of cell identity and disease. Cell 155, 
934-947 (2013).

28. Hu, Z. \& Tee, W.-W. Enhancers and chromatin structures: regulatory hubs in gene expression and diseases. Biosci. Rep. 37, BSR20160183 (2017).

29. Dias, S. et al. Effector Regulatory T Cell Differentiation and Immune Homeostasis Depend on the Transcription Factor Myb. Immunity 46, 78-91 (2017).

30. Delacher, M. et al. Genome-wide DNA-methylation landscape defines specialization of regulatory T cells in tissues. Nat. Immunol. 18, 1160-1172 (2017).

31. Hayatsu, N. et al. Analyses of a Mutant Foxp3 Allele Reveal BATF as a Critical Transcription Factor in the Differentiation and Accumulation of Tissue Regulatory $\mathrm{T}$ Cells. Immunity 47, 268-283.e9 (2017).

32. DiSpirito, J. R. et al. Molecular diversification of regulatory T cells in nonlymphoid tissues. Sci. Immunol. 3, eaat5861 (2018).

33. Sula Karreci, E. et al. Human regulatory T cells undergo self-inflicted damage via granzyme pathways upon activation. JCI insight 2, e91599 (2017).

34. Loyher, P.-L., Rodero, M. P., Combadière, C. \& Boissonnas, A. Role of Chemokines and Chemokine Receptors in Cancer. in Cancer Immunology: A Translational Medicine Context (ed. Rezaei, N.) 235-262 (Springer International Publishing, 2020). doi:10.1007/978-3-030-30845-2_14.

35. Tessema, M. et al. Differential Epigenetic Regulation of TOX Subfamily High Mobility Group Box Genes in Lung and Breast Cancers. PLoS One 7, e34850 (2012).

36. Mariotti, S. et al. MICAL2 is a novel human cancer gene controlling mesenchymal to epithelial transition involved in cancer growth and invasion. Oncotarget 7, 1808-1825 (2016). 
37. Elinav, E. et al. Inflammation-induced cancer: crosstalk between tumours, immune cells and microorganisms. Nat. Rev. Cancer 13, 759-771 (2013).

38. Verbsky, J. W. \& Chatila, T. A. Immune dysregulation, polyendocrinopathy, enteropathy, X-linked (IPEX) and IPEX-related disorders. Curr. Opin. Pediatr. 25, 708-714 (2013).

39. Parackova, Z. et al. T regulatory lymphocytes in type 1 diabetes: Impaired CD25 expression and IL-2 induced STAT5 phosphorylation in pediatric patients. Autoimmunity 49, 523-531 (2016).

40. Herrath, J. et al. The inflammatory milieu in the rheumatic joint reduces regulatory T-cell function. Eur. J. Immunol. 41, 2279-2290 (2011).

41. Petrelli, A. et al. Self-Sustained Resistance to Suppression of CD8+ Teff Cells at the Site of Autoimmune Inflammation Can Be Reversed by Tumor Necrosis Factor and Interferony Blockade. Arthritis Rheumatol. 68, 229-236 (2016).

42. Singh, R. et al. Egr2 and 3 Inhibit T-bet-Mediated IFN- $\gamma$ Production in T Cells. $J$. Immunol. 198, 4394-4402 (2017).

43. Takahashi, R., Nakatsukasa, H., Shiozawa, S. \& Yoshimura, A. SOCS1 Is a Key Molecule That Prevents Regulatory T Cell Plasticity under Inflammatory Conditions. J. Immunol. 199, 149-158 (2017).

44. Arvey, A. et al. Genetic and epigenetic variation in the lineage specification of regulatory T cells. Elife 4, e07571 (2015).

45. Gao, P. et al. Risk variants disrupting enhancers of TH1 and TREG cells in type 1 diabetes. Proc. Natl. Acad. Sci. 116, 7581-7590 (2019).

46. Beyer, M. et al. Repression of the genome organizer SATB1 in regulatory T cells is required for suppressive function and inhibition of effector differentiation. Nat. Immunol. 
12, 898-907 (2011).

47. Roychoudhuri, R. et al. BACH2 represses effector programs to stabilize Treg-mediated immune homeostasis. Nature 498, 506-510 (2013).

48. Vahedi, G. et al. Super-enhancers delineate disease-associated regulatory nodes in T cells. Nature 520, 558-562 (2015).

49. Kitagawa, Y. et al. Guidance of regulatory T cell development by Satb1-dependent superenhancer establishment. Nat. Immunol. 18, 173-183 (2017).

50. Afzali, B. et al. BACH2 immunodeficiency illustrates an association between superenhancers and haploinsufficiency. Nat. Immunol. 18, 813-823 (2017).

51. Yang, B.-H. et al. TCF1 and LEF1 Control Treg Competitive Survival and Tfr Development to Prevent Autoimmune Diseases. Cell Rep. 27, 3629-3645.e6 (2019).

52. Xing, S. et al. Tcf1 and Lef1 are required for the immunosuppressive function of regulatory T cells. J. Exp. Med. 216, 847-866 (2019).

53. Bhairavabhotla, R. et al. Transcriptome profiling of human FoxP3(+) regulatory T cells. Hum. Immunol. 77, 201-213 (2015).

54. Wienke, J. et al. Human regulatory T cells at the maternal-fetal interface show functional site-specific adaptation with tumor-infiltrating-like features [preprint]. bioRxiv 820753 (2019) doi:10.1101/820753.

55. Bscheider, M. \& Butcher, E. C. Vitamin D immunoregulation through dendritic cells. Immunology 148, 227-236 (2016).

56. Dankers, W. et al. Human Memory Th17 Cell Populations Change Into Antiinflammatory Cells With Regulatory Capacity Upon Exposure to Active Vitamin D.

Front. Immunol. 10, 1504 (2019). 
57. Meinicke, H. et al. KLRG1 impairs regulatory T-cell competitive fitness in the gut.

Immunology 152, 65-73 (2017).

58. Procaccini, C. et al. The Proteomic Landscape of Human Ex Vivo Regulatory and Conventional T Cells Reveals Specific Metabolic Requirements. Immunity 44, 406-421 (2016).

59. Vukmanovic-Stejic, M. et al. Human CD4+ CD25hi Foxp3+ regulatory T cells are derived by rapid turnover of memory populations in vivo. J. Clin. Invest. 116, 2423-2433 (2006).

60. Sun, I.-H. et al. mTOR Complex 1 Signaling Regulates the Generation and Function of Central and Effector Foxp3 + Regulatory T Cells. J. Immunol. 201, 481-492 (2018).

61. Kishore, M. et al. Regulatory T Cell Migration Is Dependent on Glucokinase-Mediated Glycolysis. Immunity 47, 875-889.e10 (2017).

62. Ferreira, L. M. R., Muller, Y. D., Bluestone, J. A. \& Tang, Q. Next-generation regulatory T cell therapy. Nat. Rev. Drug Discov. 18, 749-769 (2019).

63. Petty, R. E. et al. Revision of the proposed classification criteria for juvenile idiopathic arthritis: Durban, 1997. J. Rheumatol. 25, 1991-1994 (1998).

64. Subramanian, A. et al. Gene set enrichment analysis: a knowledge-based approach for interpreting genome-wide expression profiles. Proc. Natl. Acad. Sci. U. S. A. 102, 1554515550 (2005).

65. Ferraro, A. et al. Interindividual variation in human T regulatory cells. Proc. Natl. Acad. Sci. U. S. A. 111, E1111-1120 (2014).

66. Peeters, J. G. C. et al. Inhibition of Super-Enhancer Activity in Autoinflammatory SiteDerived T Cells Reduces Disease-Associated Gene Expression. Cell Rep. 12, 1986-1996 
(2015).

67. Robinson, J. T. et al. Integrative genomics viewer. Nature biotechnology vol. 29 24-26

(2011).

68. Whyte, W. A. et al. Master transcription factors and mediator establish super-enhancers at key cell identity genes. Cell 153, 307-319 (2013).

69. Zheng, C. et al. Landscape of Infiltrating T Cells in Liver Cancer Revealed by Single-Cell Sequencing. Cell 169, 1342-1356.e16 (2017).

70. Vishwakarma, A. et al. Mapping the Immune Landscape of Clear Cell Renal Cell Carcinoma by Single-Cell RNA-seq [preprint]. bioRxiv 824482 (2019) doi:10.1101/824482. 
Acknowledgments: We would like to thank J. van Velzen and P. Andriessen van der Burght for technical assistance. F. van Wijk is supported by a VIDI grant from ZonMw (91714332).

Author contributions: Conceptualization: G.M. and L.L., Fv.W and J.v.L. Performed experiments: G.M., L.L. M.v.d.W., R.S., M.K., S.Ve., A.P. Data analysis: G.M., L.L., M.M., M.v.d.W., J.G.C.P., A.P., W.T., and J.v.L. Patients selection and clinical interpretation: G.M., S.Va., and S.d.R. Supplied RA dataset: V.F., C.R., and L.S.T. Writing: G.M., L.L., M.v.d.W., J.v.L., and F.v.W. Supervision: J.v.L. and F.v.W. All authors reviewed and edited the manuscript.

Competing interests: The authors have declared that no competing interests exist. 
bioRxiv preprint doi: https://doi.org/10.1101/2020.09.30.319962; this version posted October 1, 2020. The copyright holder for this preprint (which was not certified by peer review) is the author/funder, who has granted bioRxiv a license to display the preprint in perpetuity. It is made available under aCC-BY-NC-ND 4.0 International license. 
bioRxiv preprint doi: https://doi.org/10.1101/2020.09.30.319962; this version posted October 1,2020. The copyright holder for this preprint (which was not certified by peer review) is the author/funder, who has granted bioRxiv a license to display the preprint in perpetuity. It is made available under aCC-BY-NC-ND 4.0 International license.

a

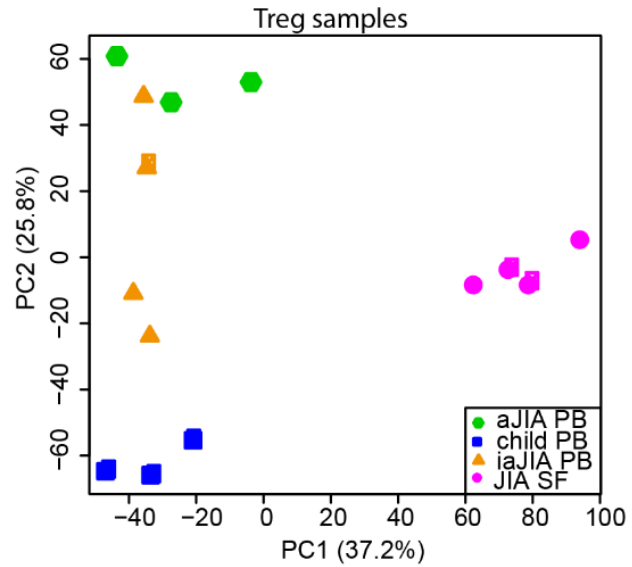

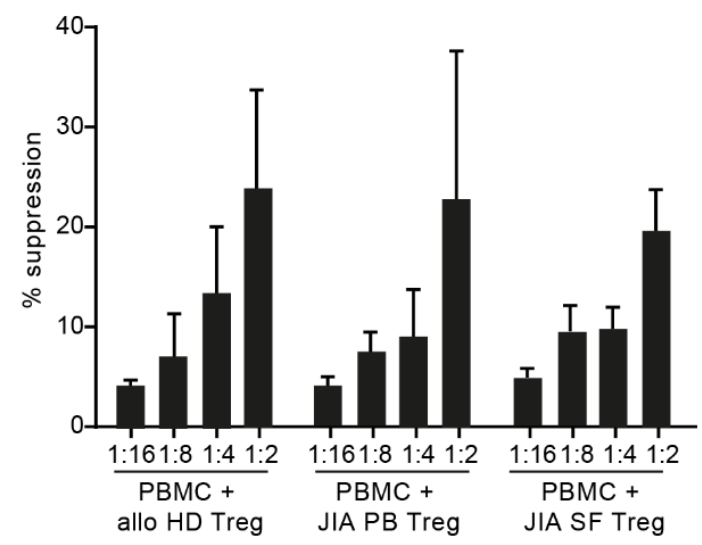

C

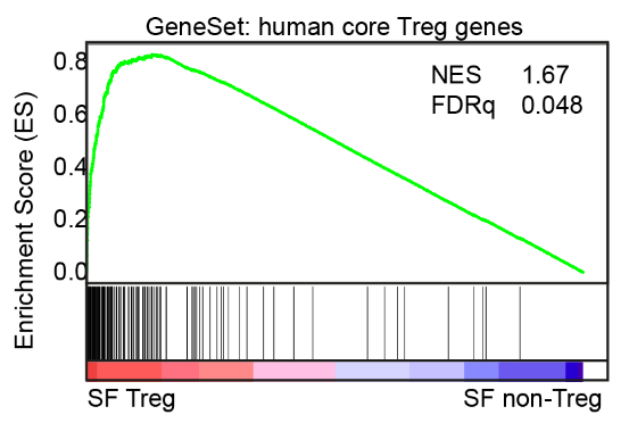

GeneSet: human core Treg genes

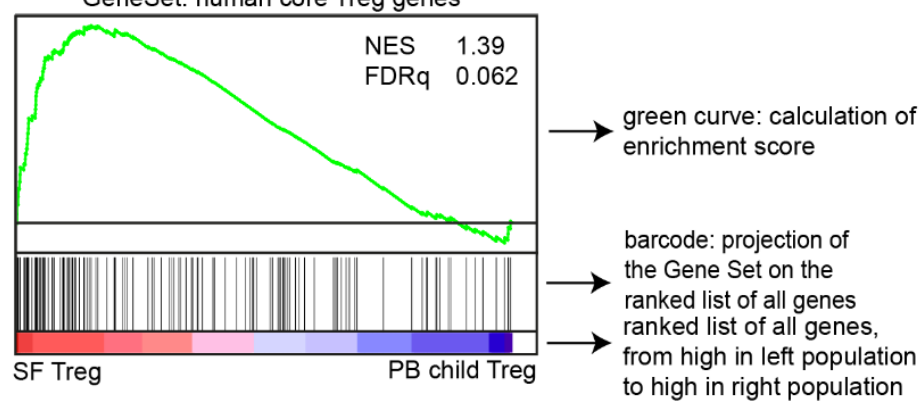

e

FOXP3
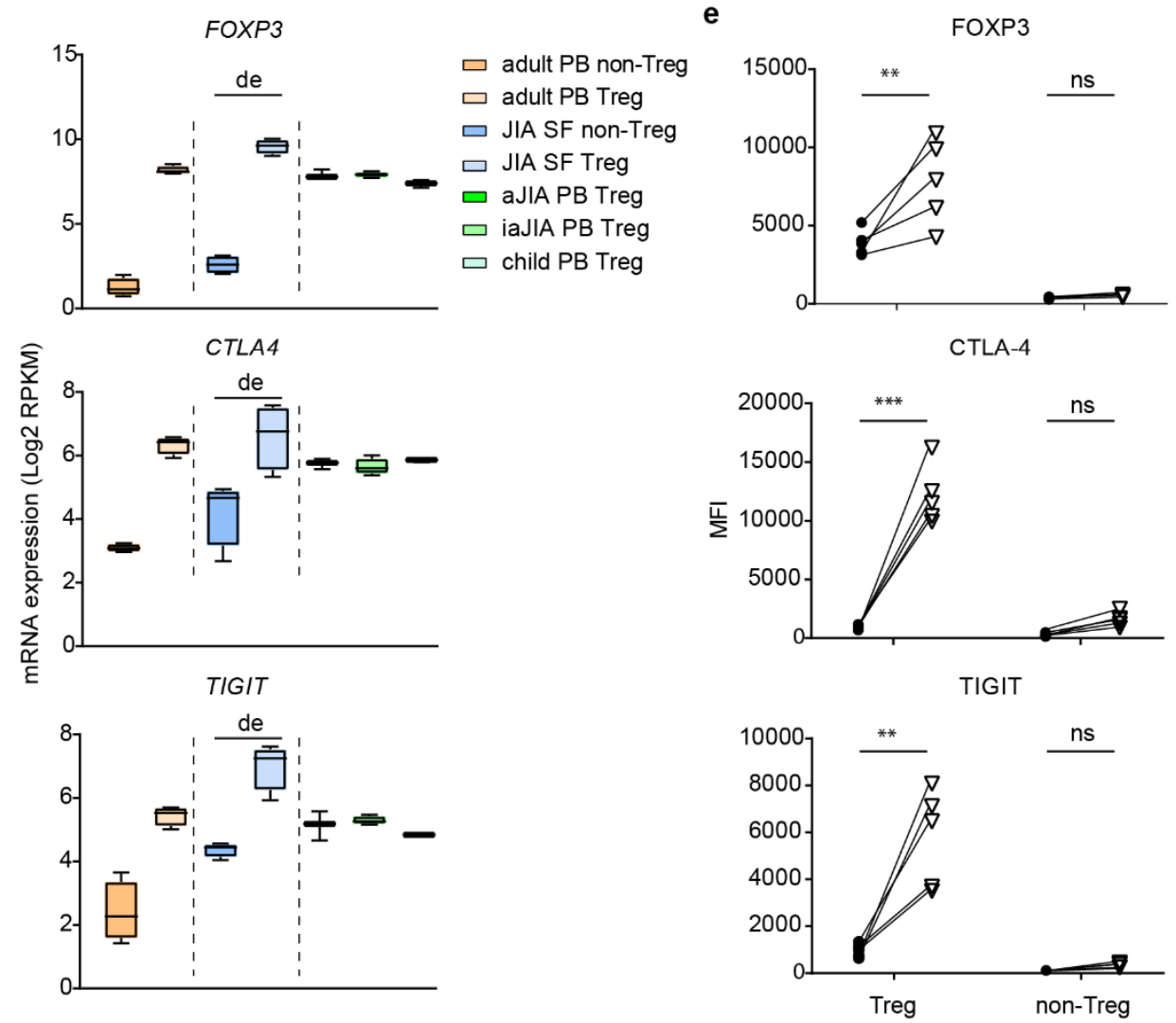

Fig. 1. Human inflammation-derived Treg are distinct from peripheral Treg with clear Treg 
characteristics. a. Unsupervised Principal Component Analysis (PCA) of all Treg groups, synovial fluid (SF)- and peripheral blood (PB)-derived, from children. b Bar charts representing suppression (percentage) of $\mathrm{CD}^{+} \mathrm{T}$ cells by Treg from $\mathrm{PB}$ of healthy donors or juvenile idiopathic arthritis (JIA) patients and SF of JIA patients. $50.000 \mathrm{ctViolet} \mathrm{labeled} \mathrm{PBMC} \mathrm{from} \mathrm{an}$ allogenic healthy donor were cultured with different ratios of Treg for 4 days in anti-CD3 coated plates $(\mathrm{n}=4$, mean + SEM). c GeneSet Enrichment Analysis (GSEA) of human core Treg signature genes (identified by Ferraro et $a l .{ }^{65}$ ) in pairwise comparisons involving SF Treg and non-Treg, and SF Treg and healthy child PB Treg, represented by the normalized enrichment score (NES) and FDR statistical value (FDRq). d mRNA expression (log2 RPKM) of FOXP3, CTLA4 and TIGIT in Treg derived from PB of healthy adults (adult), healthy children (child), JIA patients with active (aJIA) or inactive (iaJIA) disease, SF of JIA patients (JIA SF) and nonTreg from PB of healthy adults and SF of JIA patients (de = differentially expressed according to $\log 2$ fold change $\geq 0.6$, adjusted (adj)p- value $\leq 0.05$, mean of all normalized counts $>10$; adj $\mathrm{p}$ values $F O X P 3=1.1 \mathrm{E}^{-95}, C T L A 4=2.3 \mathrm{E}^{-05}, T I G I T=1.9 \mathrm{E}^{-17}$ ) e Median Fluorescence Intensity (MFI) of FOXP3, CTLA-4 and TIGIT in $\mathrm{CD}^{+} \mathrm{CD} 4^{+} \mathrm{CD} 25^{+} \mathrm{CD} 127^{\text {low }}$ Treg and $\mathrm{CD}^{+} \mathrm{CD}^{+} \mathrm{CD} 25^{-} \mathrm{CD} 127^{+}$non-Treg from paired SFMC and PBMC from 5 JIA patients. Statistical comparisons were performed using two-way ANOVA with Sidak correction for multiple testing: ns, $* * P<0.01, * * * P<0.001$. (b and $\mathbf{e})$ Data are representative of two independent experiments. 
bioRxiv preprint doi: https://doi.org/10.1101/2020.09.30.319962; this version posted October 1,2020. The copyright holder for this preprint (which was not certified by peer review) is the author/funder, who has granted bioRxiv a license to display the preprint in perpetuity. It is made available under aCC-BY-NC-ND 4.0 International license.

a

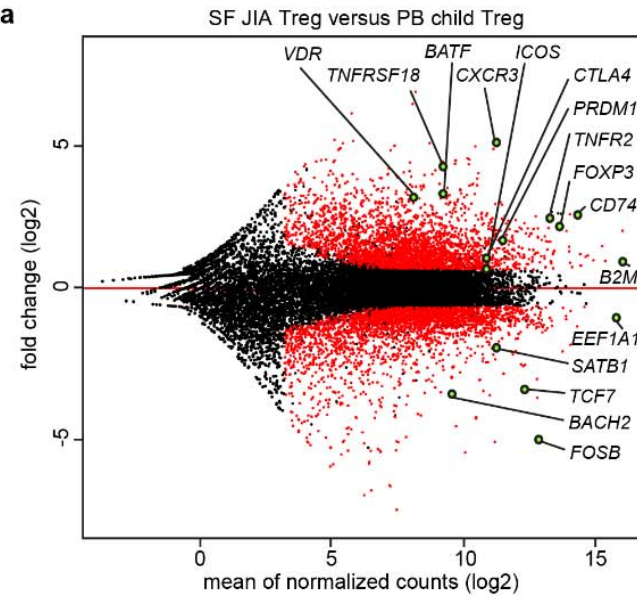

c
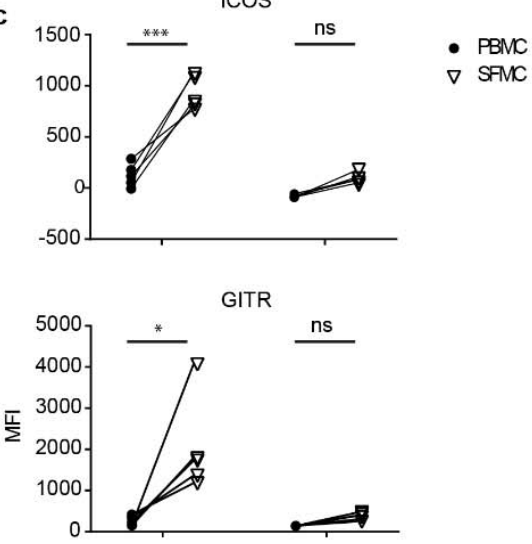

PD-1
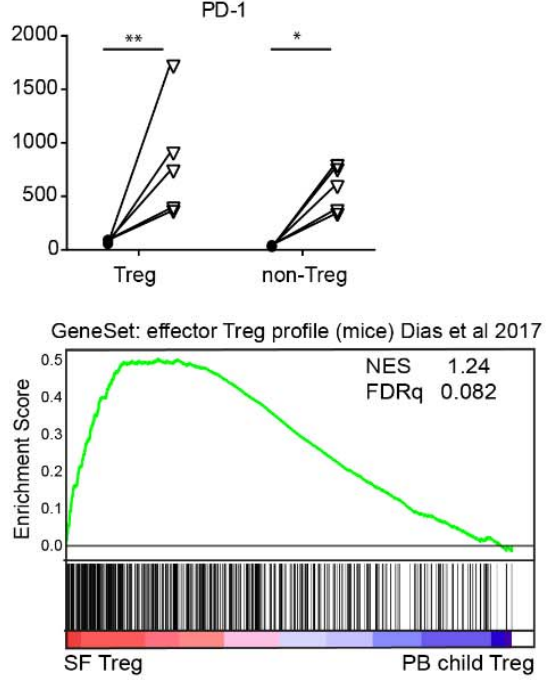

b

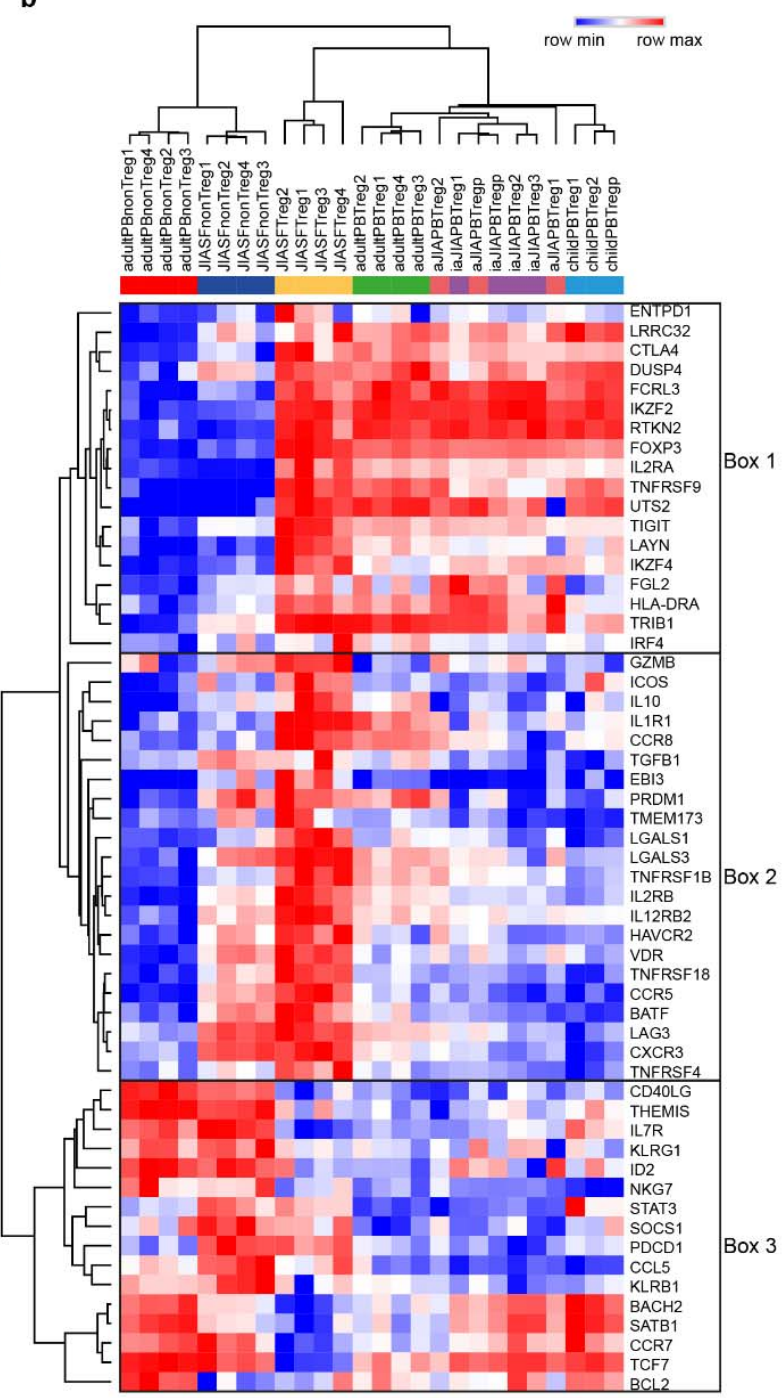

Fig. 2. Inflammatory environment-derived Treg demonstrate a specific effector profile. a MA plot of the differentially expressed genes between synovial fluid (SF) and peripheral blood (PB) Treg of healthy children with black dots reflecting no change, and red dots transcripts with an 
adjusted p-value $<0.05$, minimal mean of all normalized counts $>10$ and log2 fold change $>0.6$. b Heatmap with hierarchical clustering analysis including all groups measured with RNAsequencing selected genes based on recent literature (relative expression of log2 RPKM). c MFI of ICOS, GITR and PD-1 in gated $\mathrm{CD} 3^{+} \mathrm{CD} 4^{+} \mathrm{CD} 25^{+} \mathrm{CD} 127^{\text {low }}$ Treg and $\mathrm{CD} 3^{+} \mathrm{CD} 4^{+} \mathrm{CD} 25^{-}$ $\mathrm{CD}_{127^{+}}$non-Treg from paired SFMC and PBMC from 5 JIA patients. Statistical comparisons were performed using two-way ANOVA with Sidak correction for multiple testing: ns, ${ }^{*} P<$ $0.05, * * P<0.01, * * * P<0.001$. Data are representative of two or more independent experiments. d GeneSet Enrichment Analysis (GSEA) of effector Treg genes in mice (identified by Dias et $a l .{ }^{29}$ ) in pairwise comparisons involving SF Treg and PB Treg derived from healthy children, represented by the normalized enrichment score (NES) and FDR statistical value (FDRq). 
a

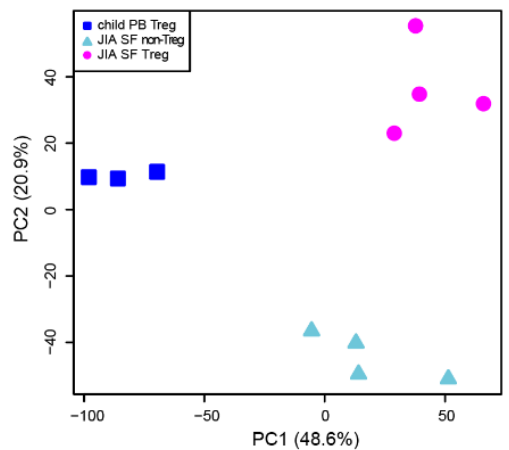

c

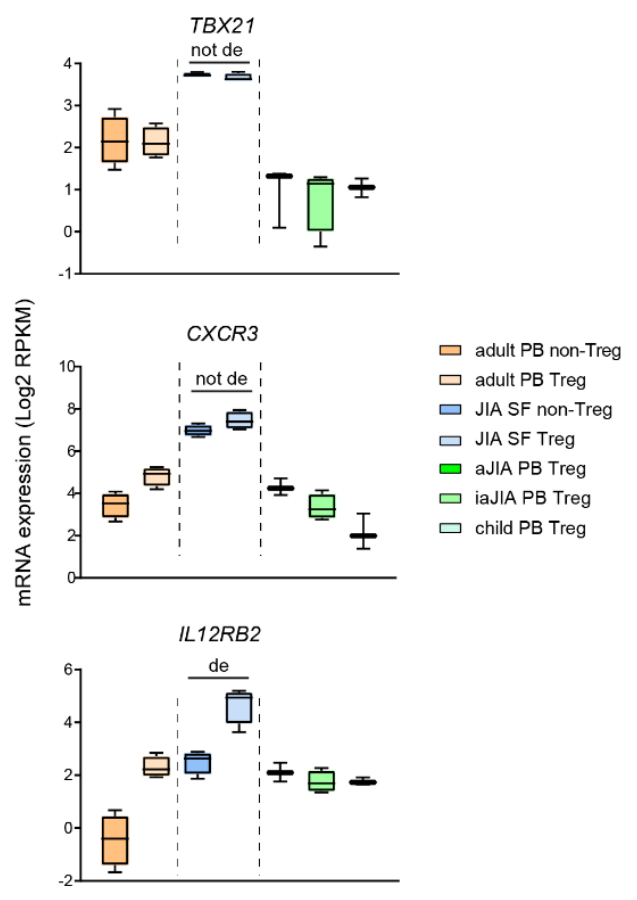

e

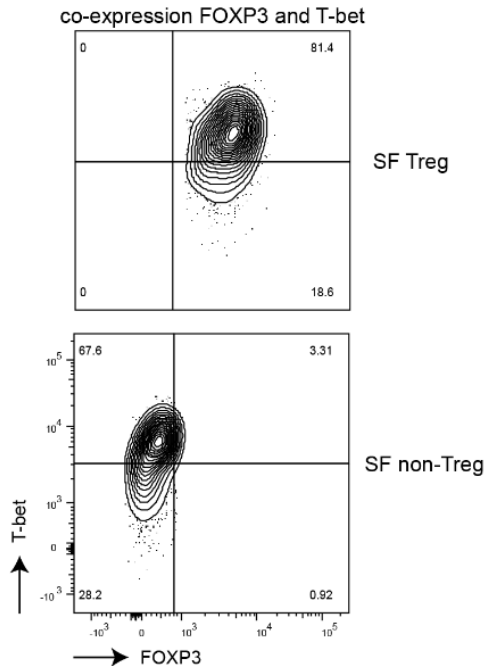

b
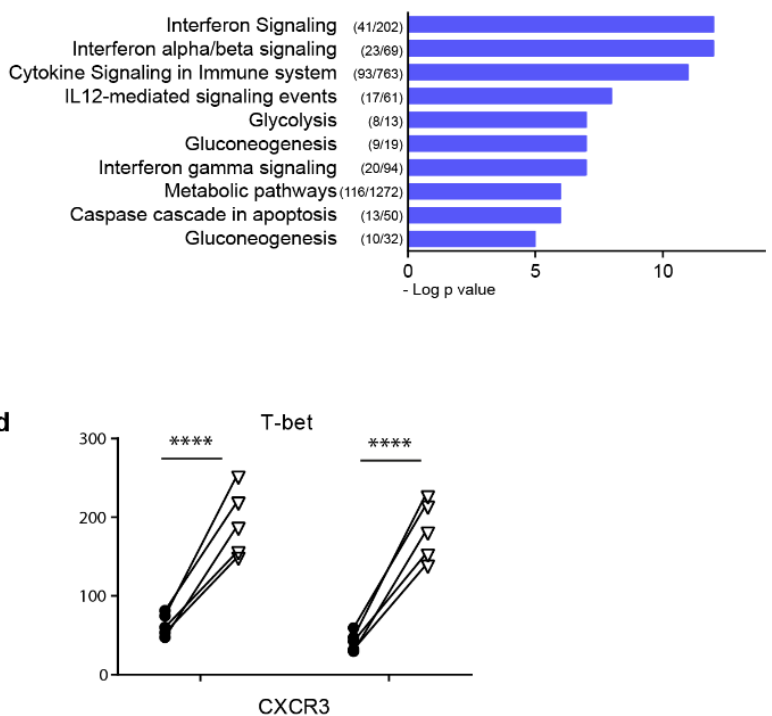

CXCR3
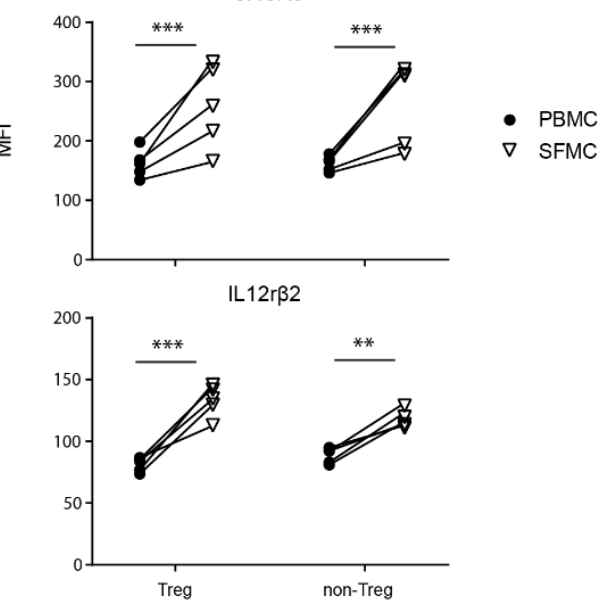

f

IFNy

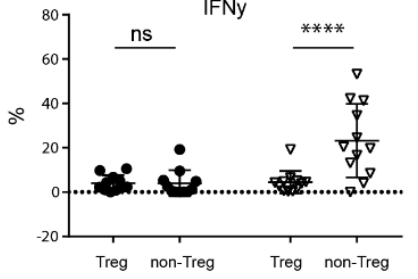

- PB

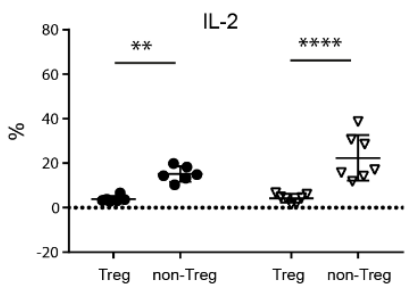

Fig. 3. SF Treg adapt to the interferon-skewed inflammatory environment but lack cytokine production. a Unsupervised Principal Component Analysis (PCA) of synovial fluid (SF)-derived 
Treg and non-Treg and peripheral blood (PB)-derived Treg from healthy children. b Gene ontology terms related to the 1791 genes specifically upregulated in SF compared to PB Treg derived from children (active juvenile idiopathic arthritis (JIA), inactive JIA and healthy children), ranked by enrichment scores. The number of upregulated genes compared to the total number annotated in the gene ontology term are depicted before the terms. c mRNA expression ( $\log 2 \mathrm{RPKM})$ of $T B X 21, C X C R 3$ and $I L 12 R B 2$ in Treg derived from PB of healthy adults (adult), healthy children (child), JIA patients with active (aJIA) or inactive (iaJIA) disease, SF of JIA patients (JIA SF) and non-Treg from PB of healthy adults and SF of JIA patients (de = differentially expressed according to description in Fig. 1d). d MFI of T-bet, CXCR3 and IL12R $\beta 2$ in $\mathrm{CD}^{+} \mathrm{CD} 4^{+} \mathrm{CD} 25^{+} \mathrm{CD} 127^{\text {low }}$ Treg and $\mathrm{CD} 3^{+} \mathrm{CD} 4^{+} \mathrm{CD} 25^{-} \mathrm{CD} 127^{+}$non-Treg from paired SFMC and PBMC from 5 JIA patients. e Representative contourplot of T-bet and FOXP3 in $\mathrm{CD}^{+} \mathrm{CD}^{+} \mathrm{CD} 25^{+} \mathrm{FOXP}^{+}$Treg and $\mathrm{CD}^{+} \mathrm{CD}^{+} \mathrm{CD} 25^{\text {int/- }}$ FOXP3 $3^{-}$non-Treg from SFMC. $\mathbf{f}$ Percentage of IFN $\gamma$ and $\mathrm{IL}-2$ measured in $\mathrm{CD} 3^{+} \mathrm{CD} 4^{+} \mathrm{CD} 25^{+} \mathrm{FOXP} 3^{+}$Treg and $\mathrm{CD}^{+}{ }^{+} \mathrm{CD} 4^{+} \mathrm{CD} 25^{\text {int/-}} \mathrm{FOXP}^{-}$non-Treg from SFMC and PMBC (IFN $\gamma: \mathrm{n}=11 \mathrm{~PB}, \mathrm{n}=12 \mathrm{SF}$; IL2: $n=6 \mathrm{~PB}, \mathrm{n}=7 \mathrm{SF}$ ). (d and f) Data are representative of two independent experiments. Statistical comparisons were performed using two-way ANOVA with Sidak correction for multiple testing. ns, $* P<0.05, * * P<0.01, * * * * P<0.0001$. 
a
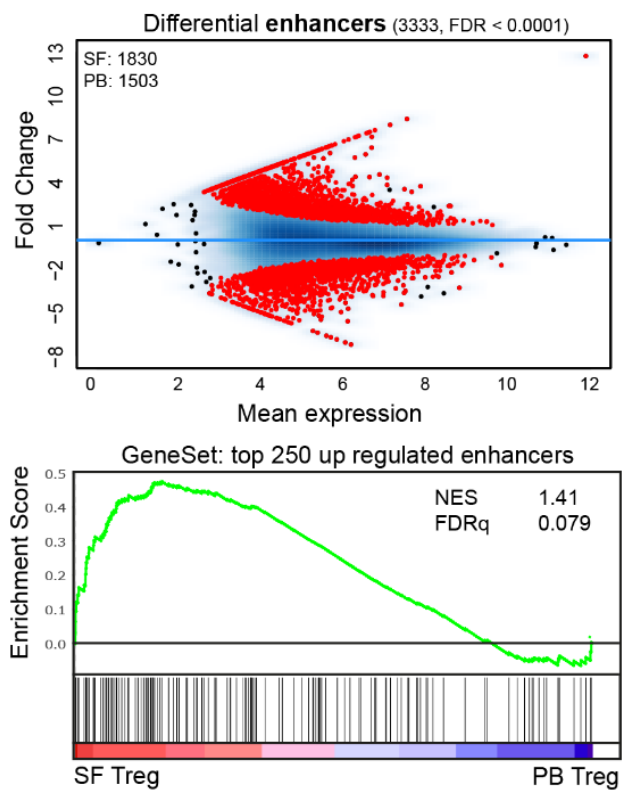

GeneSet: top 250 down regulated enhancers

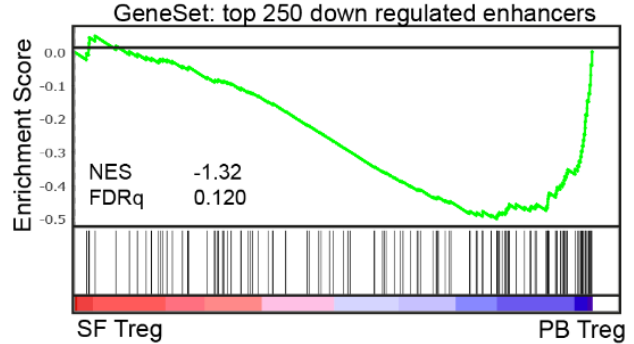

C

\begin{tabular}{ccccc}
\multicolumn{5}{c}{ DE super-enhancers } \\
up & \multicolumn{2}{c}{ down } \\
\hline BATF & ID2 & RXRA & BACH2 & ITGA6 \\
BCL2L1 & IL10 & SOCS1 & BIRC3 & KLRG1 \\
CCND2 & IL12RB2 & SRGN & CCR7 & MAML2 \\
CCR5 & KAT2B & TBX21 & CD44 & NFKB1 \\
CCR8 & LGALS3 & TNFRSF18 & CD69 & S1PR1 \\
CD58 & MAF & TNFRSF8 & CXCR4 & SATB1 \\
CTLA4 & MIIAL2 & TNIP1 & DOCK9 & SMAD3 \\
FAM129A & NAB1 & TOX2 & DPH5 & SPEN \\
FURIN & PDCD1 & TRAF3 & EDAR & TCF7 \\
HLA-DQA1 & PFKFB3 & TRIB1 & IL6ST & TGFBR2 \\
ICOS & PHLDA1 & VDR & IL7R & TRIB2
\end{tabular}

e

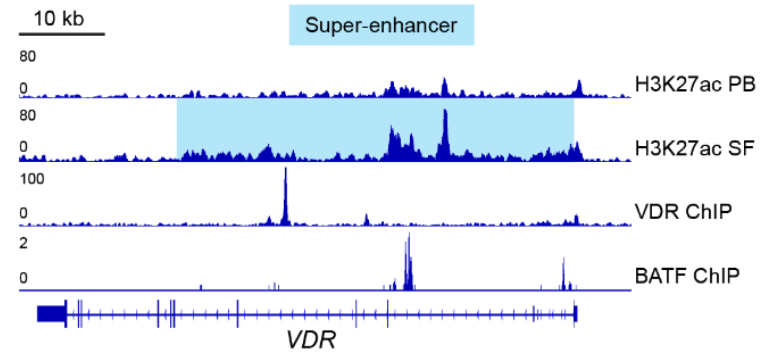

b
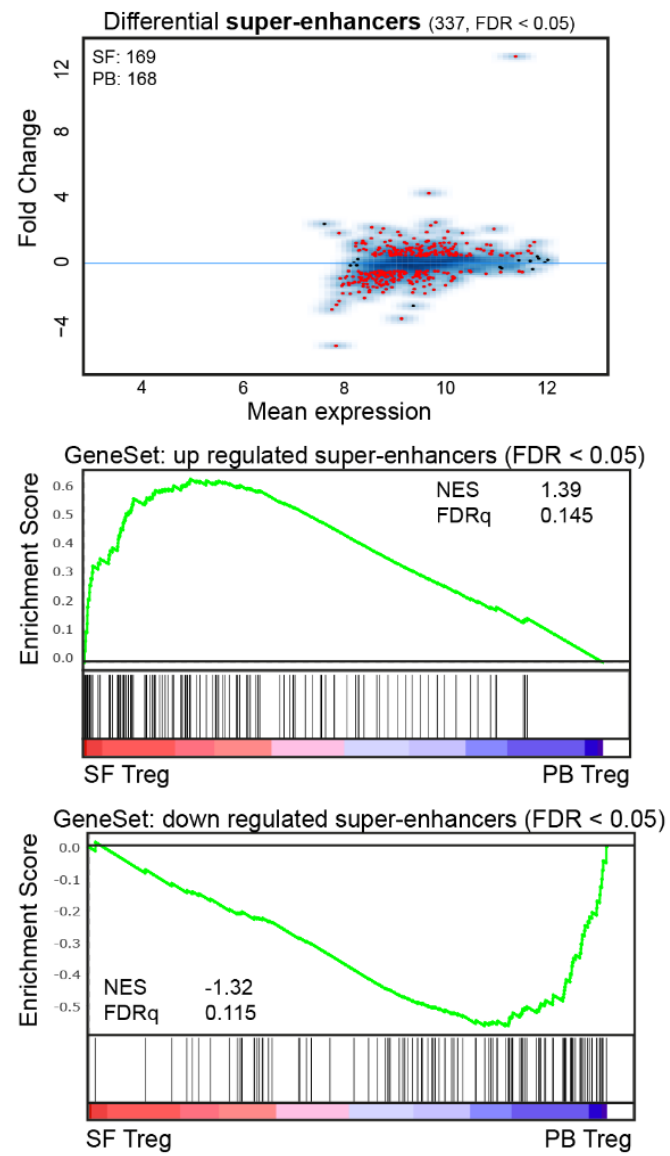

d

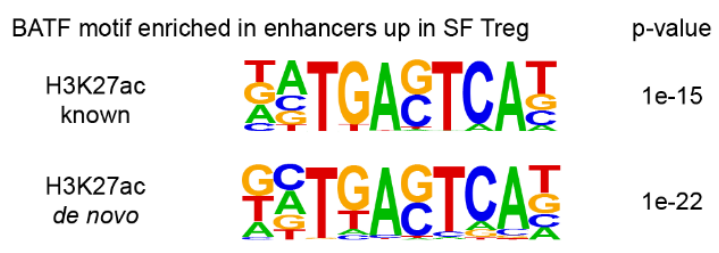

VDR motif enriched in super-enhancers up in SF Treg

H3K4me1
de novo

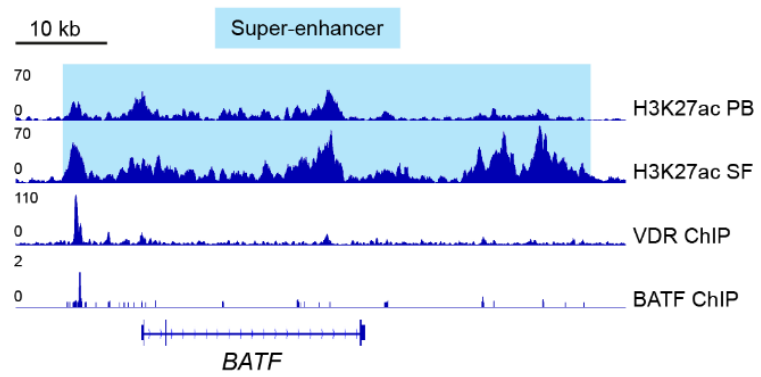

Fig. 4. Environment-specific effector Treg profile is regulated by the (super-)enhancer landscape. a MA plots of differentially expressed enhancers $($ FDR $<0.0001)$ in synovial fluid (SF) versus peripheral blood (PB) Treg for H3K27ac ChIP-seq with the number of SF- and PB- 
specific enhancers indicated (top). GeneSet Enrichment Analysis (GSEA) of the top 250 upregulated (middle) and downregulated (bottom) enhancers in pairwise comparisons involving transcriptome data of SF Treg and healthy adult PB Treg, represented by the normalized enrichment score (NES) and FDR statistical value (FDRq). b Same as in a but for superenhancers (FDR < 0.05). c Selection of super-enhancers up- and downregulated in SF Treg versus healthy adult PB Treg for H3K27ac and H3K4me1 ChIP-seq (FDR $<0.05$; bold $=$ up/down for both and differentially expressed in SF versus PB Treg on transcriptome level, see also Supplementary Table 2). d Motifs, known and de novo, for transcription factor binding sites predicted using HOMER, enriched in the upregulated (super-)enhancers in SF Treg compared to healthy adult PB Treg for H3K27ac and H3K4me1 ChIP-seq. e Gene tracks for VDR and BATF (H3K27ac) displaying ChIP-seq signals, with the super-enhancer region highlighted in blue, in healthy adult PB Treg, SF Treg, VDR-specific (GSE89431) and a BATF-specific (GSE32465) ChIP-seq. 
bioRxiv preprint doi: https://doi.org/10.1101/2020 09.30.319962. this version posted October 1 2020. The copyright holder for this preprint (which was not certified by peer review) is the author/funder, who has granted bioRxiv a license to display the preprint in perpetuity. It is made available under aCC-BY-NC-ND 4.0 International license.

a

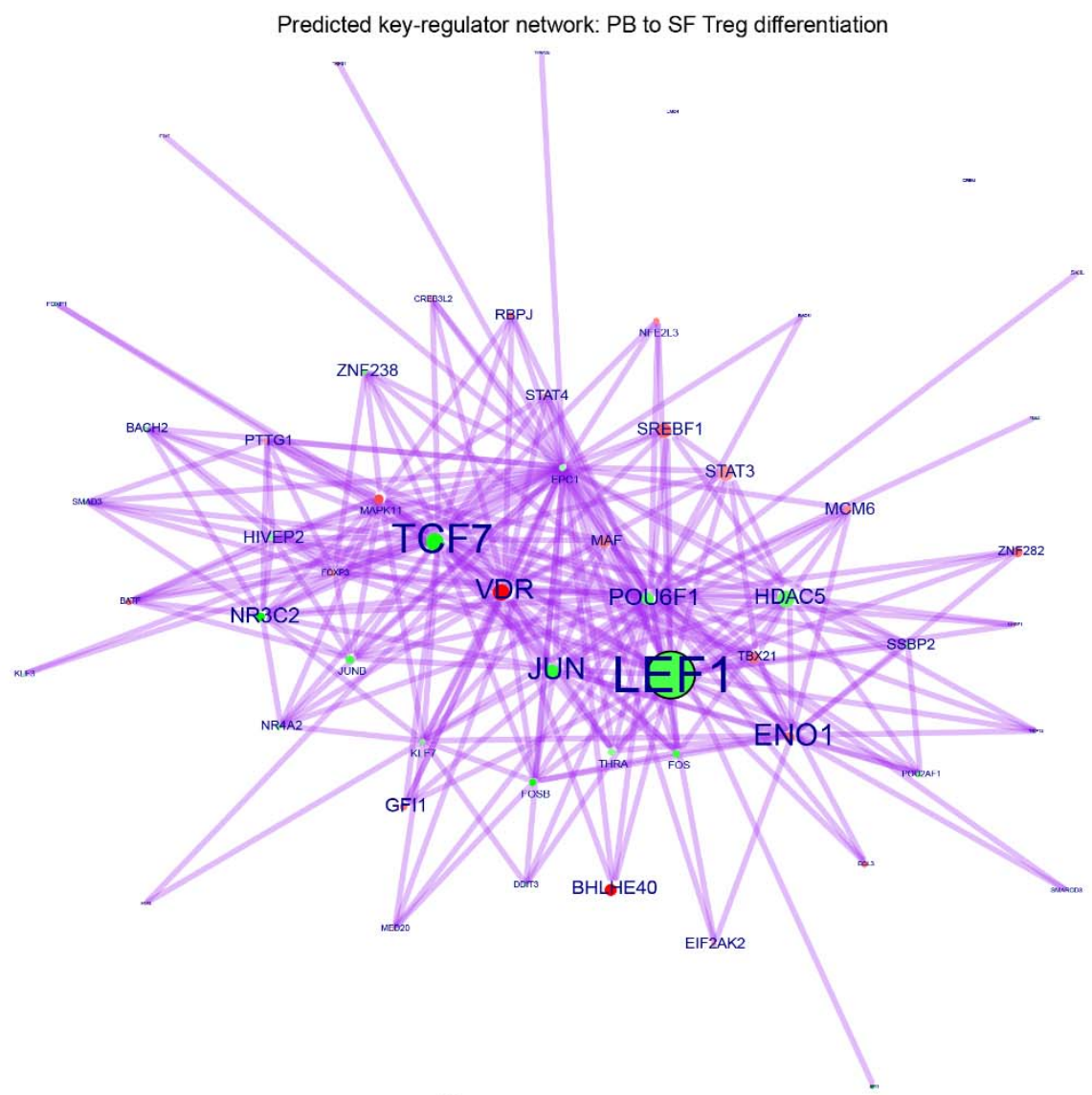

b

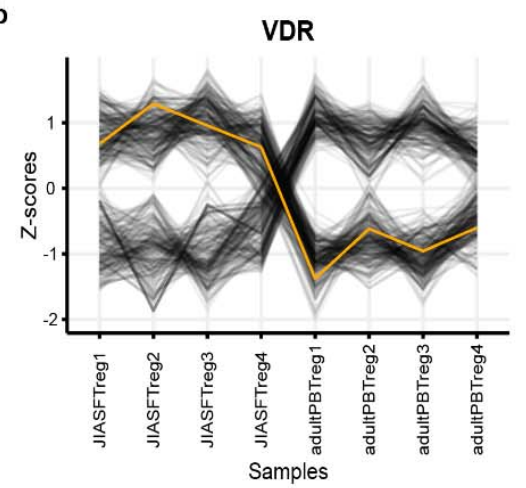

c

\begin{tabular}{ccc}
\multicolumn{3}{c}{ VDR-associated genes } \\
\hline POU6F1 & MAF & TCF3 \\
MLXIP & HSBP1 & JUNB \\
POLR1D & ZFPM1 & FOSB \\
ZBTB25 & SREBF1 & SCAF1 \\
MAX & PHF12 & TRIM28 \\
FOS & NF1 & ZNF341 \\
BCL11B & THRA & SLA2 \\
MTA1 & STAT3 & MAPK11 \\
ZNF174 & HDAC5 & FOXP3 \\
CREBBP & TBX21 & PIAS1 \\
SRCAP & SMURF2 & ZNF263
\end{tabular}

d
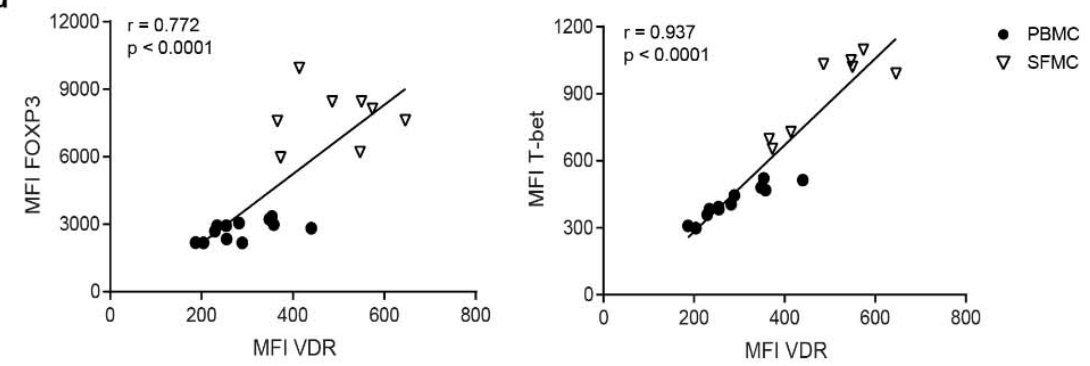

Fig. 5. The vitamin D receptor is a predicted key-regulator of effector Treg differentiation. a Network inference of key-regulators driving peripheral blood (PB) to synovial fluid (SF) Treg 
differentiation on RNA level, based on unsupervised weighted correlation network analysis followed by Fisher's exact test (red $=$ upregulation, green $=$ downregulation). Circle size indicates $-\log 10(\mathrm{p})$ for each comparison, with the $\mathrm{p}(-\mathrm{value})$ derived from differential expression analysis (log fold change > 1), and the text size represents the RegEnrich score; for both, larger indicates higher scores (see also Supplementary Table 3). b The RNA expression profile of VDR from JIA SF Treg samples 1-4 to adult PB Treg samples 1-4 indicated by the yellow line, with its associated genes in grey; values are normalized to the Z-score. c All genes associated with VDR defined by the key-regulator network inference. d Pearson's correlation plot of VDR and FOXP3 (left) and T-bet (right) MFI's in PB $(\bullet)$ and SF $(\boldsymbol{\nabla})$ with the line fitted by linear regression $(\mathrm{n}=$ $12 \mathrm{~PB} \mathrm{HC}$ and PB JIA (PBMC), $\mathrm{n}=8 \mathrm{SF}$ JIA (SFMC)). Data are representative of two independent experiments. 
bioRxiv preprint doi: https://doi.org/10.1101/2020.09.30.319962; this version posted October 1 2020. The copyright holder for this preprint (which was not certified by peer review) is the author/funder, who has granted bioRxiv a license to display the preprint in perpetuity. It is made available under aCC-BY-NC-ND 4.0 International license.

a

Expression signature genes in Treg from Rheumatoid Arthritis patients

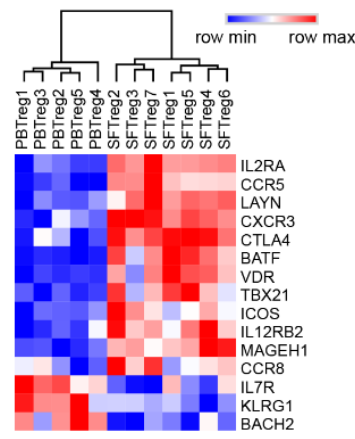

C

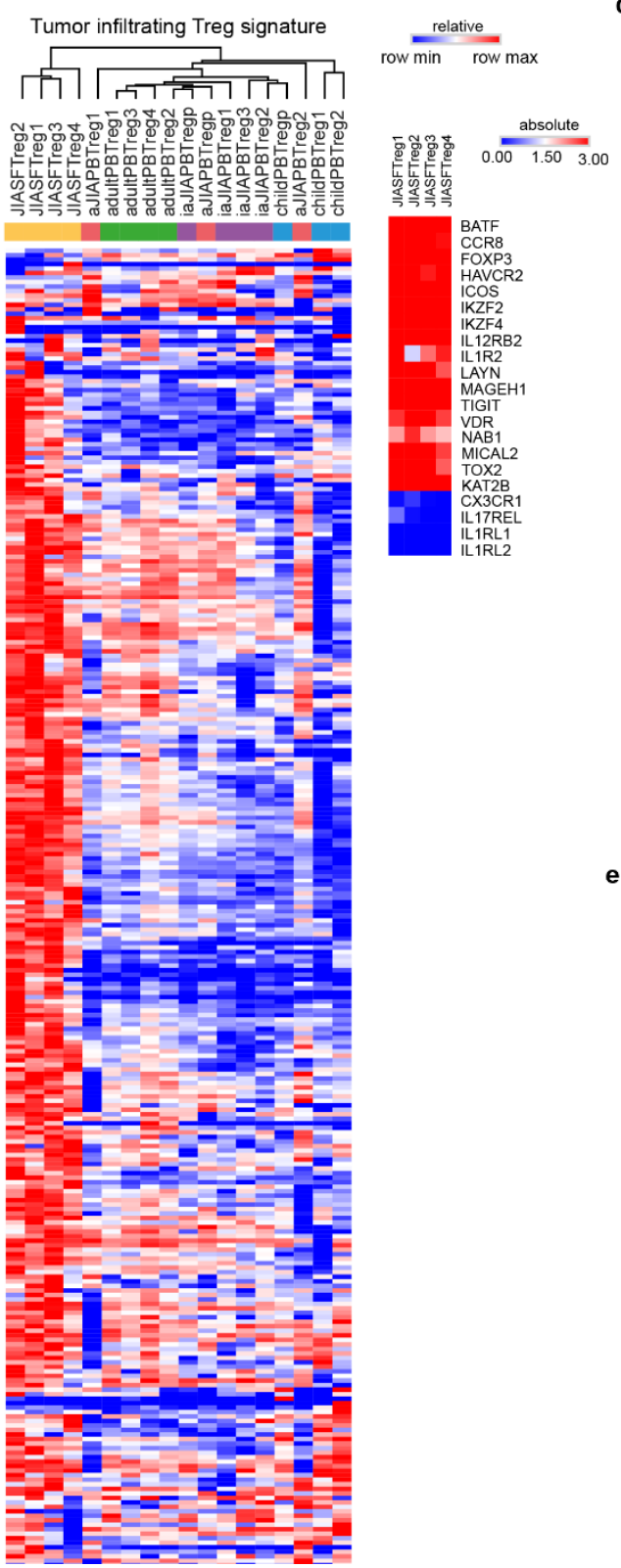

b

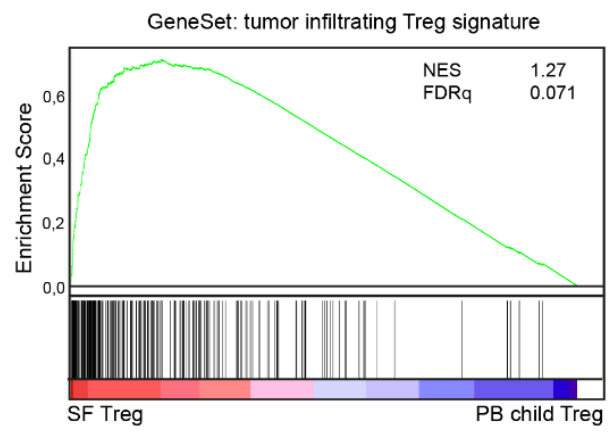

d

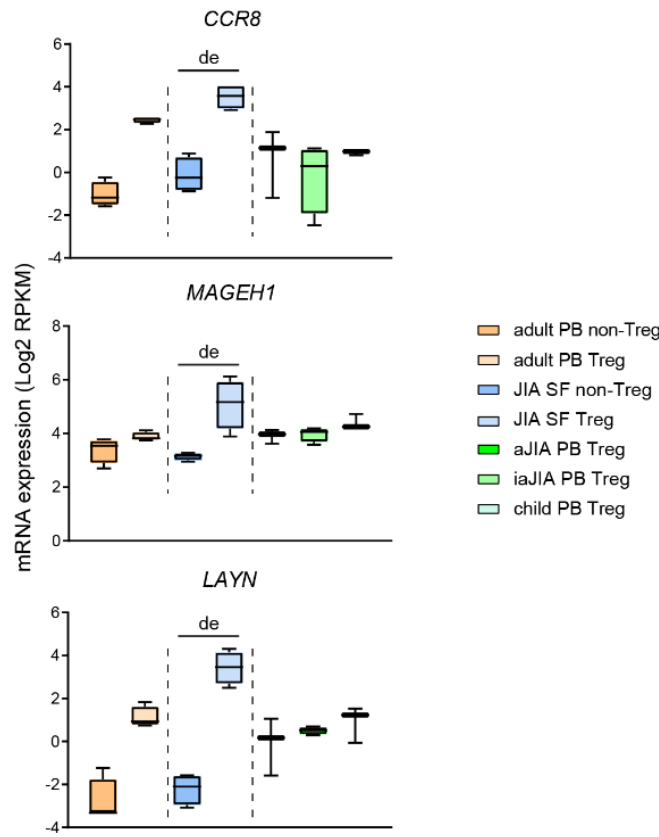

\begin{tabular}{ccc}
\multicolumn{3}{c}{ shared eTreg signature } \\
\hline ARPP19 & HNRNPLL & PFKFB3 \\
BATF & ICOS & PHLDA1 \\
BCL2L1 & IL12RB2 & RBPJ \\
CCND2 & IL1R1 & SH2D2A \\
CCR8 & IL1R2 & SRGN \\
CD58 & KAT2B & TMEM173 \\
CTSC & LAIR2 & TNFRSF18 \\
ENTPD1 & LGALS3 & TNFRSF1B \\
FAM129A & MAF & TOX2 \\
GAPDH & MICAL2 & VDR \\
GCNT1 & NAMPT &
\end{tabular}

Fig. 6. The effector Treg program is universal and overlaps with the human tumor-infiltrating

Treg signature. a Heatmap with unsupervised hierarchical clustering analysis on peripheral blood 
(PB) and (partially paired) synovial fluid (SF) Treg from Rheumatoid Arthritis patients measured with a gene array, on selected signature genes identified from juvenile idiopathic arthritis (JIA) SF Treg and tumor-infiltrating Treg. b GeneSet Enrichment Analysis (GSEA) of tumorinfiltrating Treg signature genes (identified by De Simone et al. ${ }^{13}$ ) in pairwise comparisons involving SF Treg and healthy child PB Treg, represented by the normalized enrichment score (NES) and FDR statistical value (FDRq). c Heatmap with hierarchical clustering analysis including all groups measured with RNA-sequencing on the identified tumor-infiltrating Treg signature genes (ref. ${ }^{13}$ ), with relative expression of $\log 2$ RPKM. Small heatmap on the right shows $\log 2$ RPKM values of a selection of genes in SF Treg. d mRNA expression (log2 RPKM) of CCR8, MAGEH1 and $L A Y N$ in Treg from PB of healthy adults (adult), healthy children (child), JIA patients with active (aJIA) or inactive (iaJIA) disease, SF of JIA patients (JIA SF) and non-Treg from PB of healthy adults and SF of JIA patients as determined by RNAsequencing analysis $(\mathrm{de}=$ differentially expressed according to description in Fig. 1d; adjusted pvalues $\left.C C R 8=2.2 \mathrm{E}^{-13}, M A G E H 1=1.2 \mathrm{E}^{-07}, L A Y N=1.2 \mathrm{E}^{-33}\right)$. e Identification of a human effector Treg profile based on the overlapping genes upregulated in JIA SF Treg, RA SF Treg and tumor-infiltrating Treg (identified in several studies, see refs. ${ }^{13-15,69,70}$ ), and reflected on the (super-)enhancer landscape of SF Treg. 
bioRxiv preprint doi: https://doi org/10.1101/2020.09 30.319962; this version posted October 1, 2020. The copyright holder for this preprint

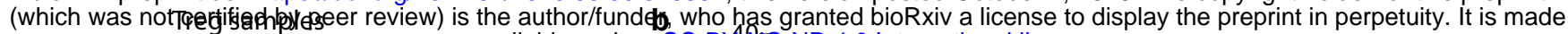
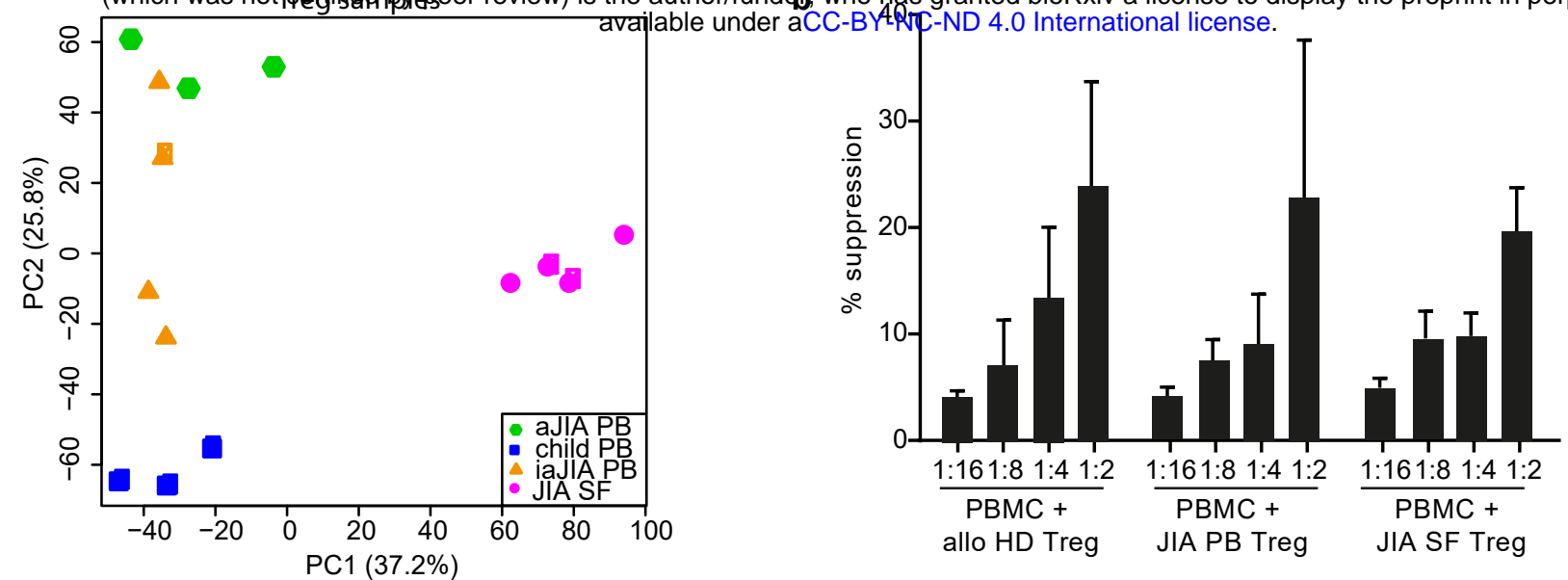

C
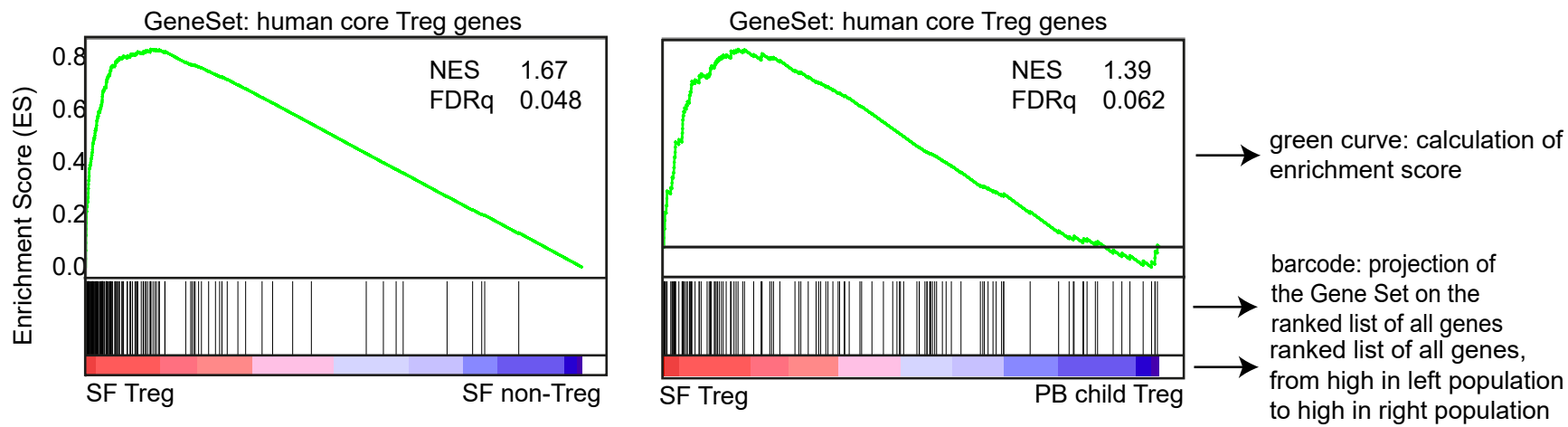

d
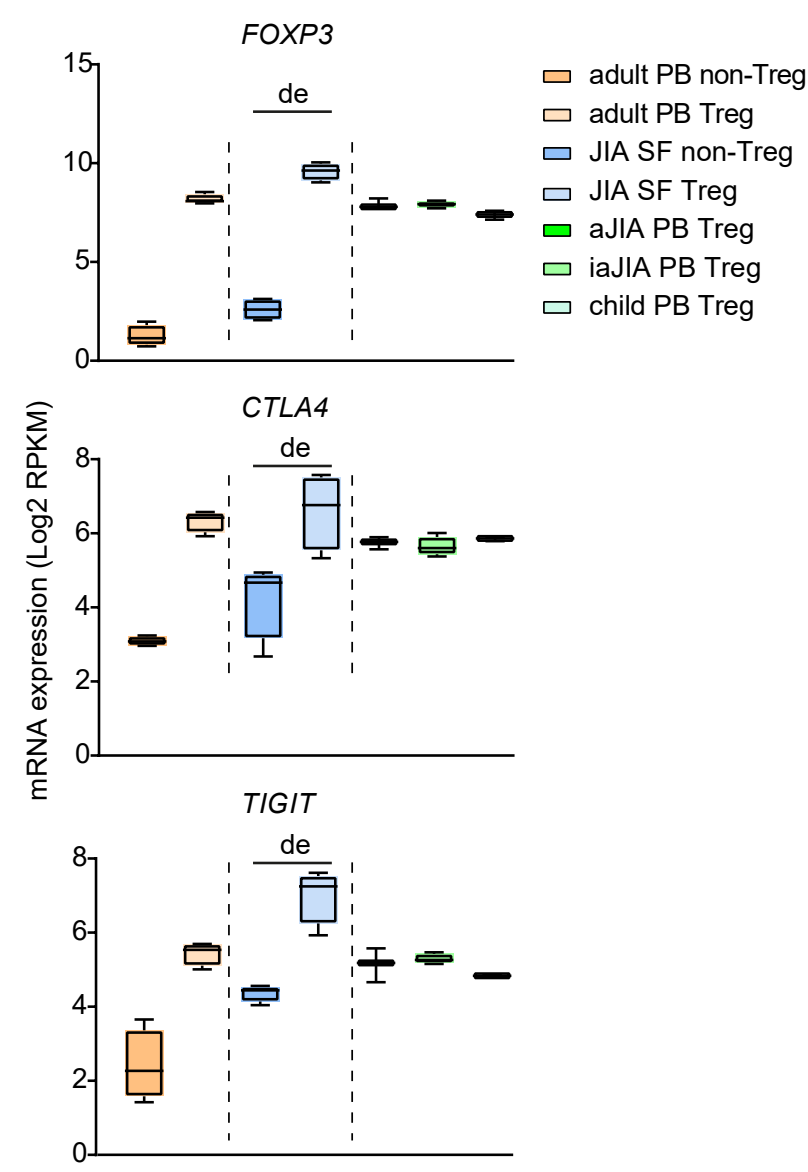

e

FOXP3

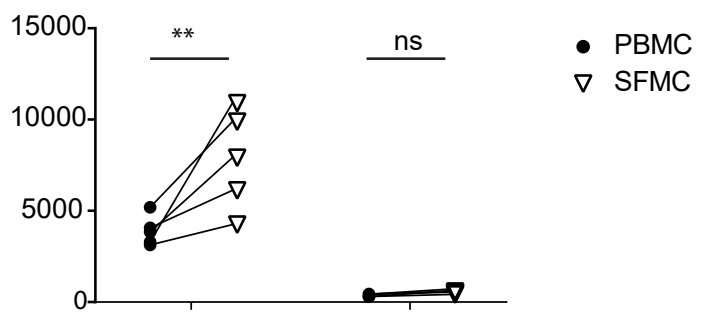

CTLA-4

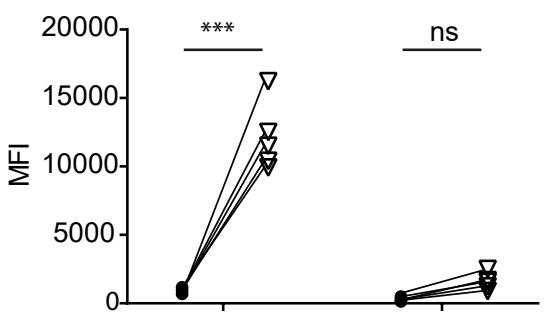

TIGIT

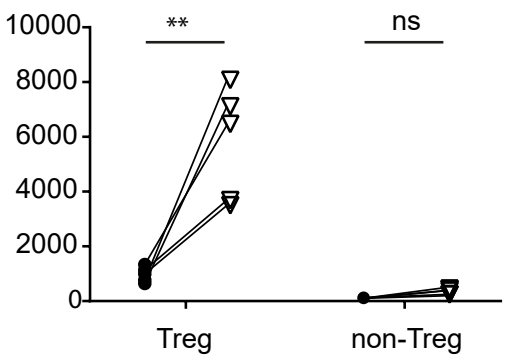


bioRxiv preprint doi: https://doi.org/10.1101/2020.09.30.319962; this version posted October 1, 2020. The copyright holder for this preprint (which was not certified by peer review) is the author/funder, who has granted bioRxiv a license to display the preprint in perpetuity. It is made SF JIA Treg versus PB child Tagilable under aCb-BY-NC-ND 4.0 International license.
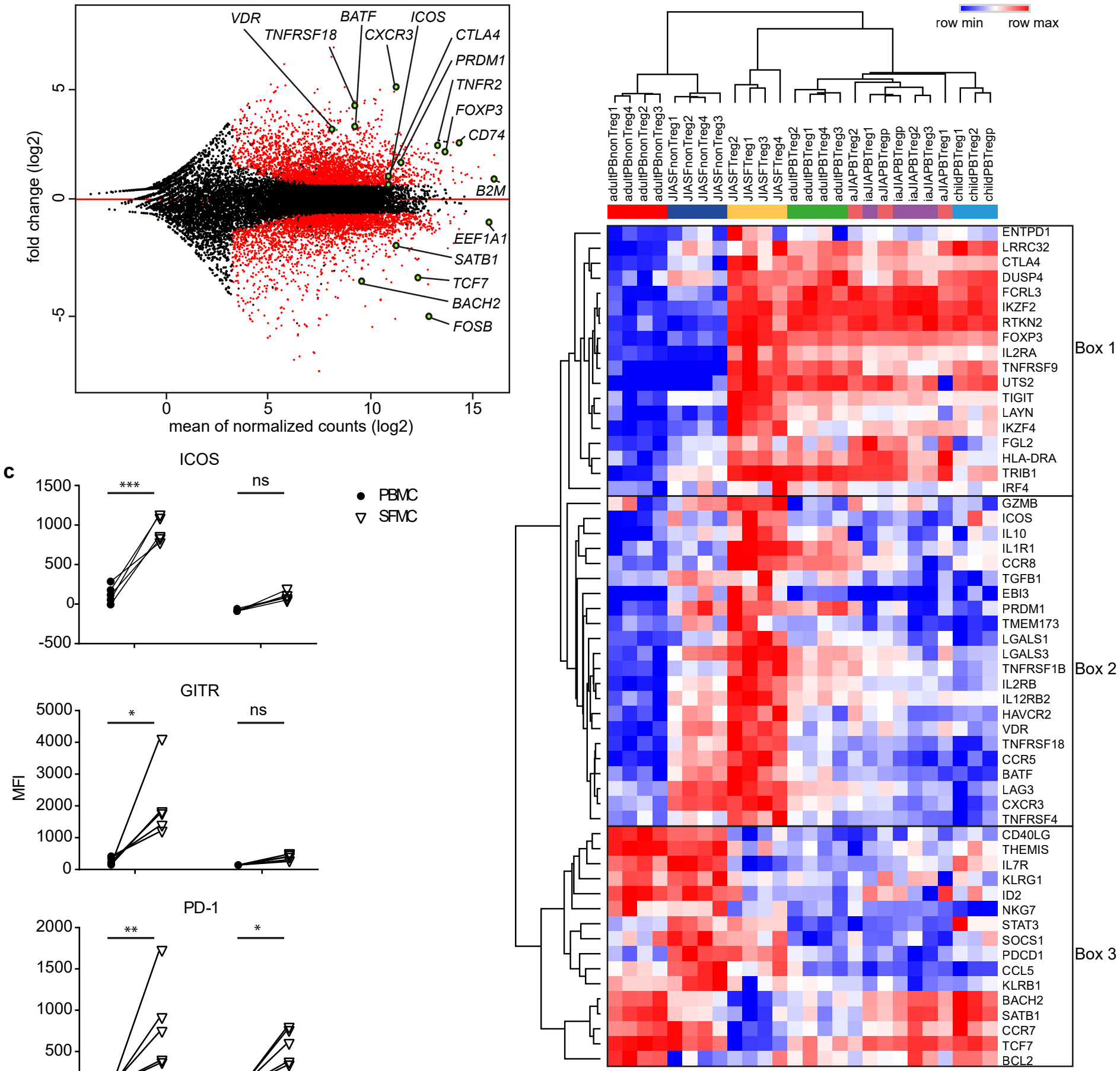

- $\mathrm{PBMC}$

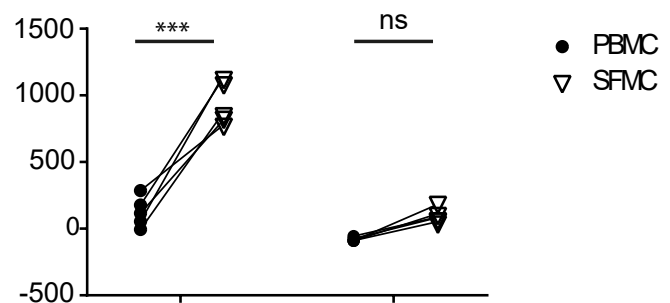

PD-1

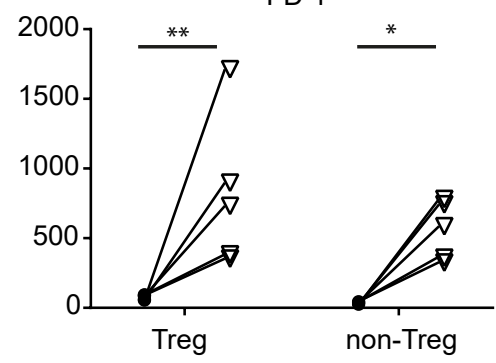

GeneSet: effector Treg profile (mice) Dias et al 2017

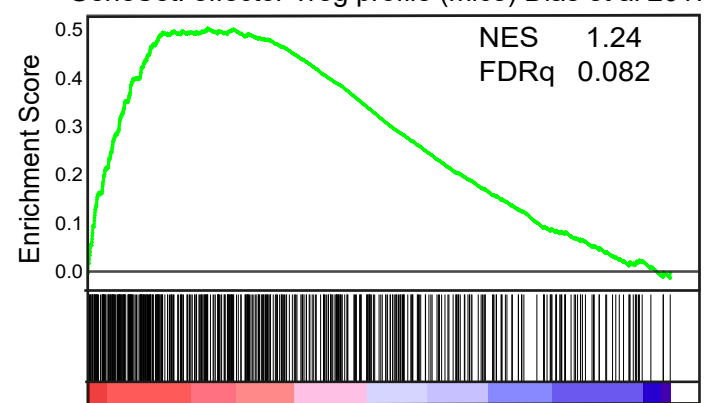

SF Treg

PB child Treg 
bioRxiv preprint doi: https://doi.org/10.1101/2020.09.30.319962; this version posted October 1, 2020. The copyright holder for this preprint

a

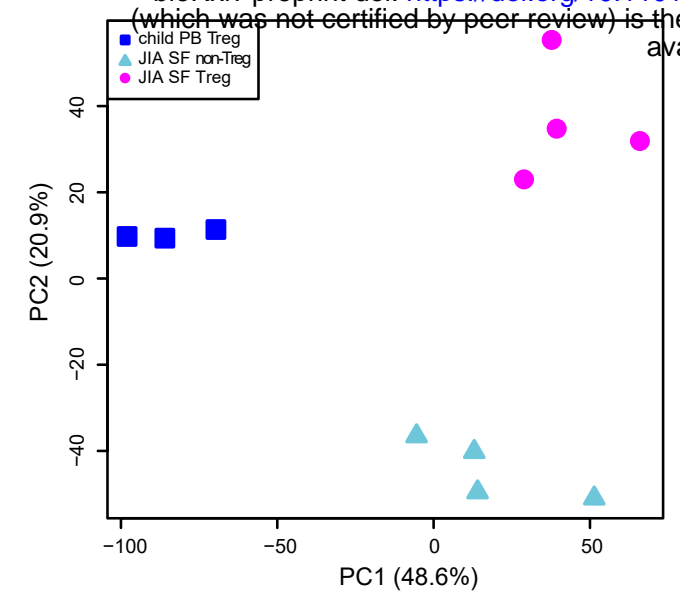

C
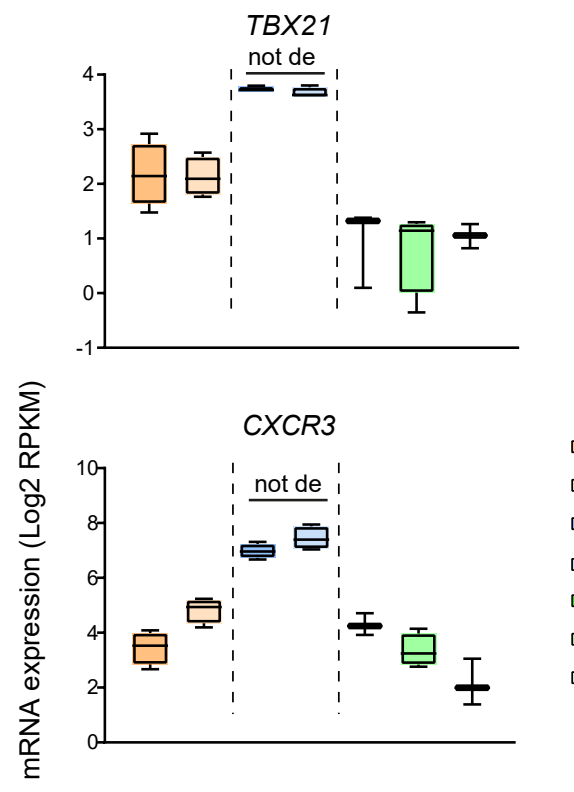

$\square$ adult PB non-Treg

$\square$ adult PB Treg

$\square$ JIA SF non-Treg

$\square$ JIA SF Treg

$\square$ aJIA PB Treg

$\square$ iaJIA PB Treg

$\square$ child PB Treg e

co-expression FOXP3 and T-bet
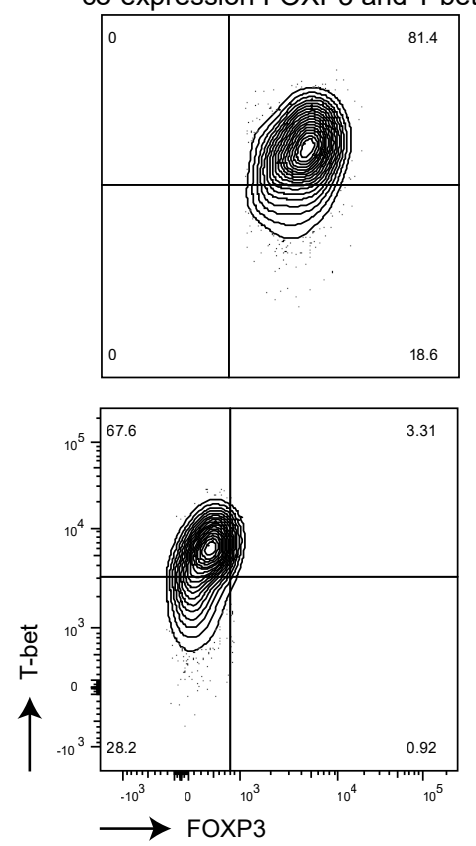

Interferon Signaling (41/202) Interferon alpha/beta signaling Cytokine Signaling in Immune system

IL12-mediated signaling events Glycolysis Gluconeogenesis Interferon gamma signaling Metabolic pathways $(116 / 1272)$

Caspase cascade in apoptosis Gluconeogenesis

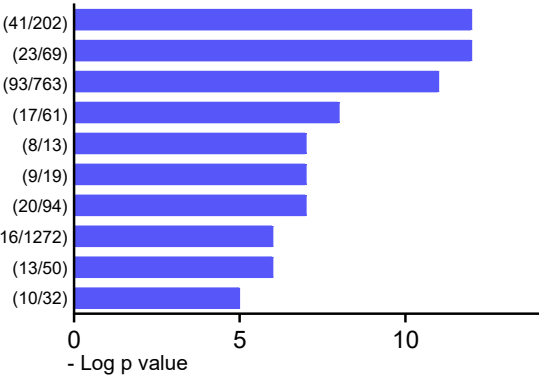

d
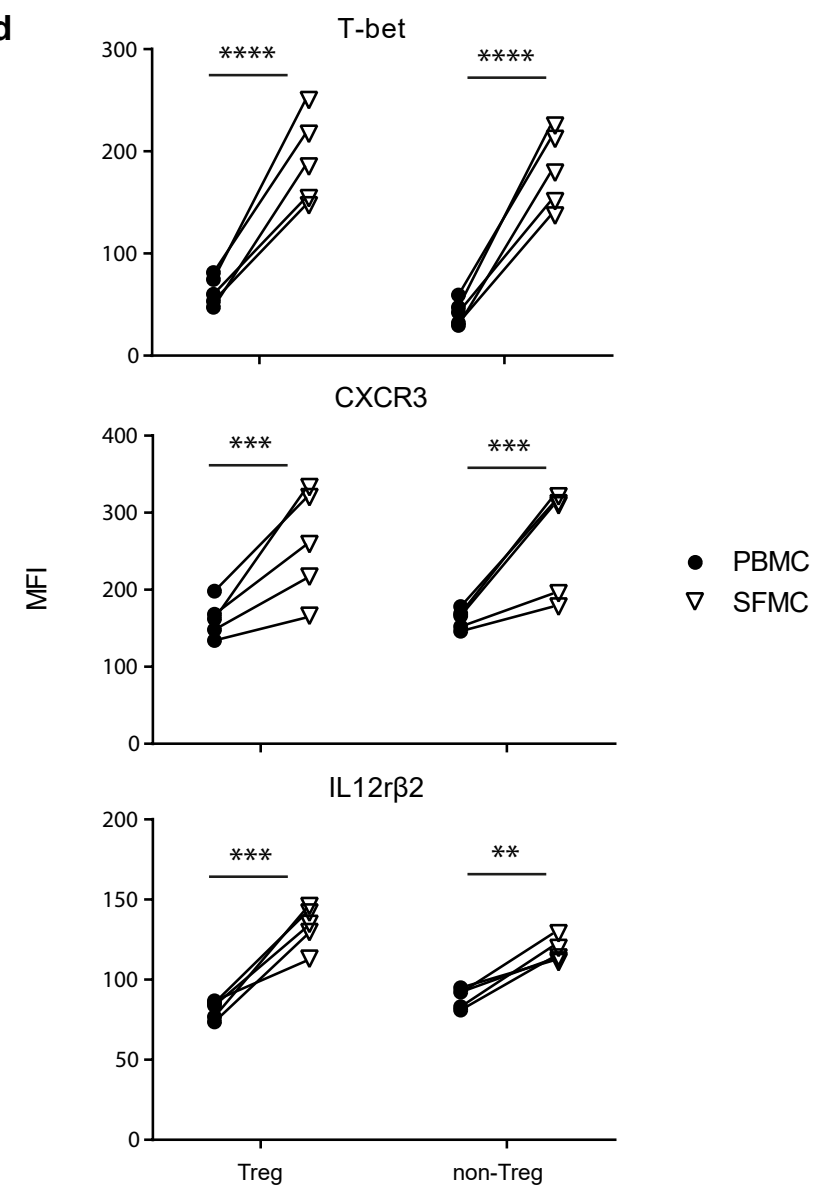

f

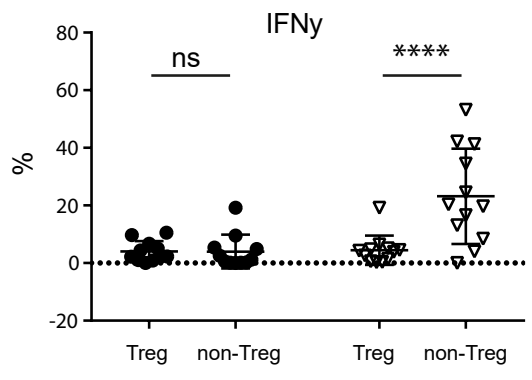

- PB

$\nabla$ SF

SF non-Treg

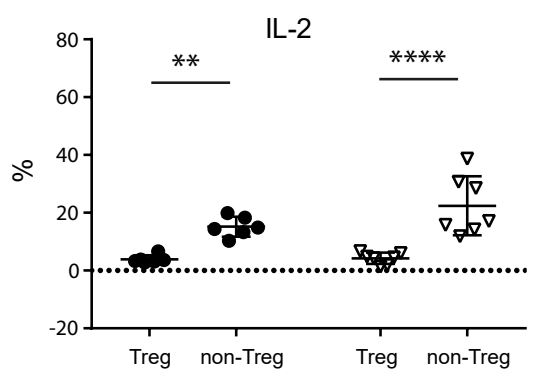


bioRxiv preppint doi: https://doi.org/10.1101/2020.09.30.319962; this version posted October 1, 2020. The copyright holder for this preprint

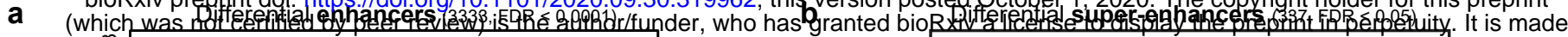
SF: $1830 \quad$ available under aCC-BY-NC-ND 4.0 lnt essational license.
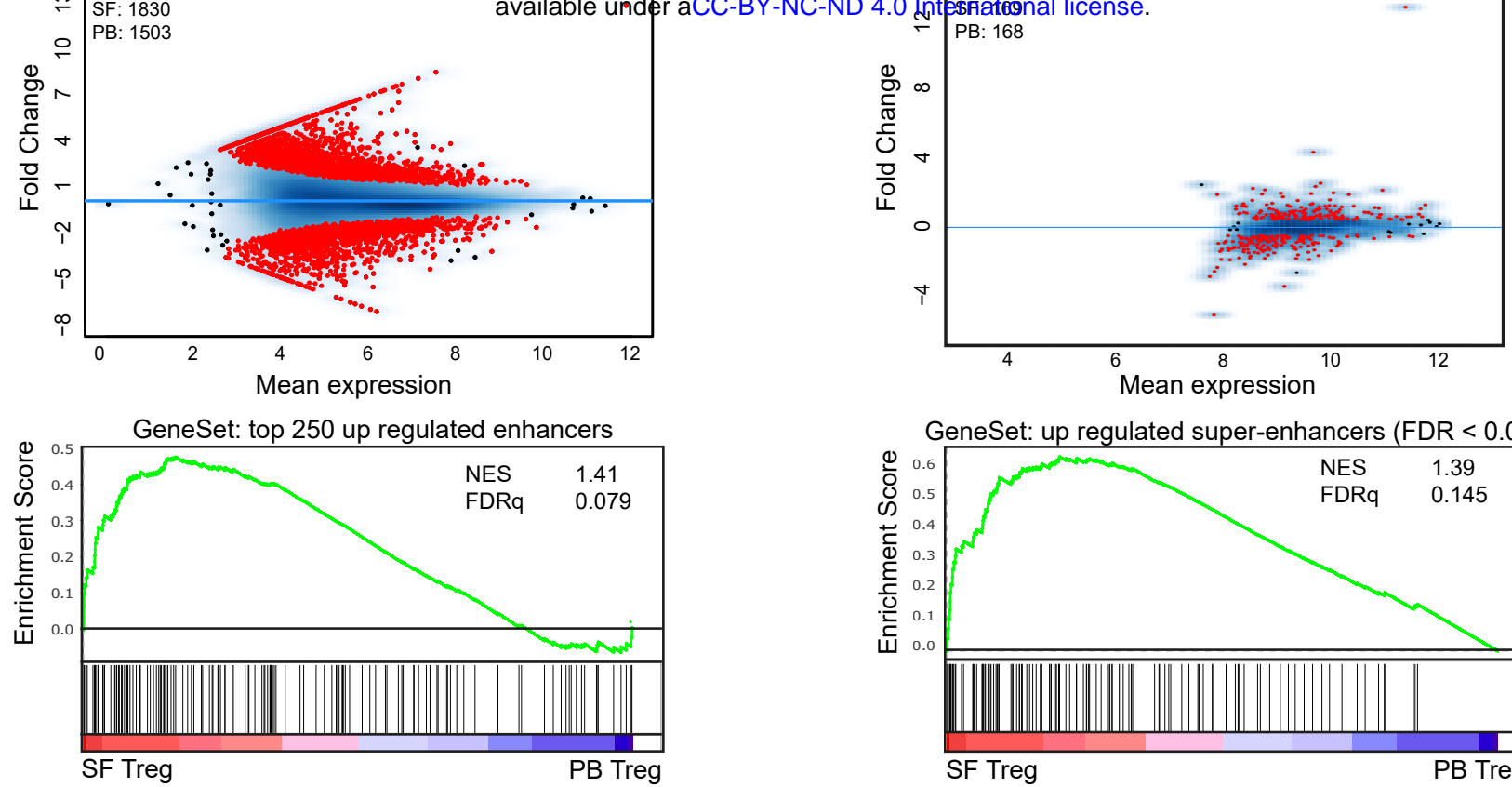

GeneSet: up regulated super-enhancers (FDR $<0.05$ )
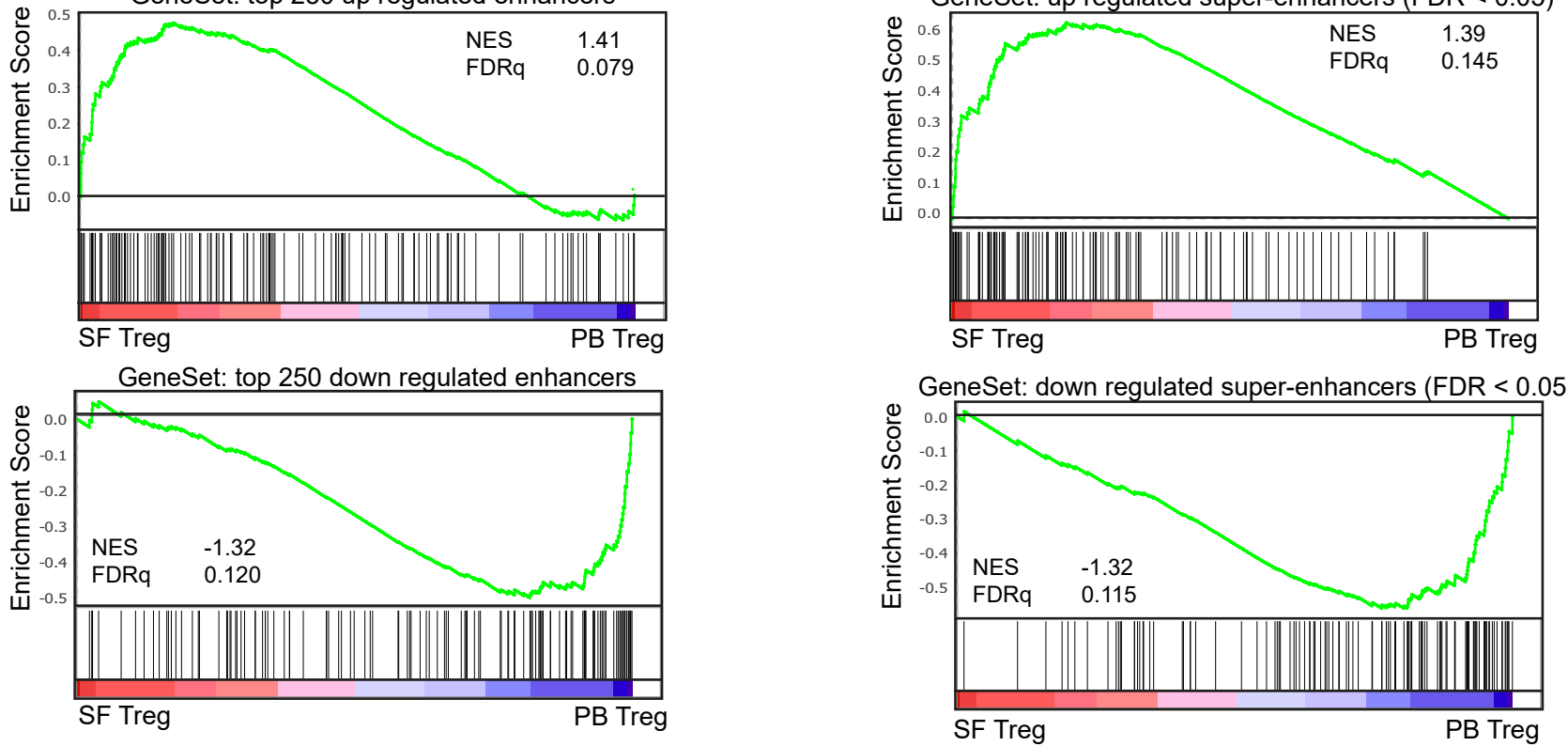

\begin{tabular}{ccc:cc}
\multicolumn{5}{c}{ DE super-enhancers } \\
up & & \multicolumn{2}{c}{ down } \\
\hline BATF & ID2 & RXRA & BACH2 & ITGA6 \\
BCL2L1 & IL10 & SOCS1 & BIRC3 & KLRG1 \\
CCND2 & IL12RB2 & SRGN & CCR7 & MAML2 \\
CCR5 & KAT2B & TBX21 & CD44 & NFKB1 \\
CCR8 & LGALS3 & TNFRS18 & CD69 & S1PR1 \\
CD58 & MAF & TNFRSF8 & CXCR4 & SATB1 \\
CTLA4 & MICAL2 & TNIP1 & DOCK9 & SMAD3 \\
FAM129A & NAB1 & TOX2 & DPH5 & SPEN \\
FURIN & PDCD1 & TRAF3 & EDAR & TCF7 \\
HLA-DQA1 & PFKFB3 & TRIB1 & IL6ST & TGFBR2 \\
ICOS & PHLDA1 & VDR & IL7R & TRIB2 \\
& & & &
\end{tabular}

e

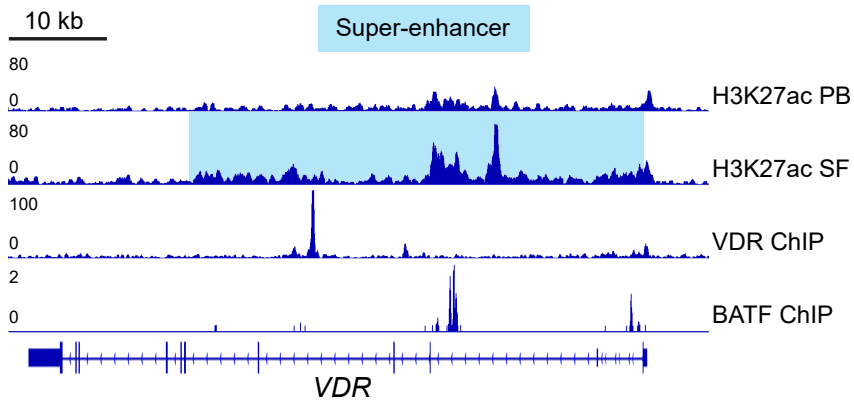

d

BATF motif enriched in enhancers up in SF Treg

$\begin{aligned} & \text { H3K27ac } \\ & \text { known }\end{aligned}$
$\begin{gathered}\text { H3K27ac } \\ \text { de novo }\end{gathered}$

VDR motif enriched in super-enhancers up in SF Treg

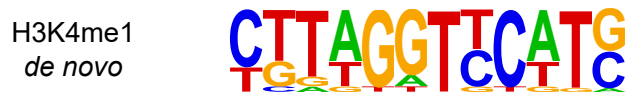
1 e-19

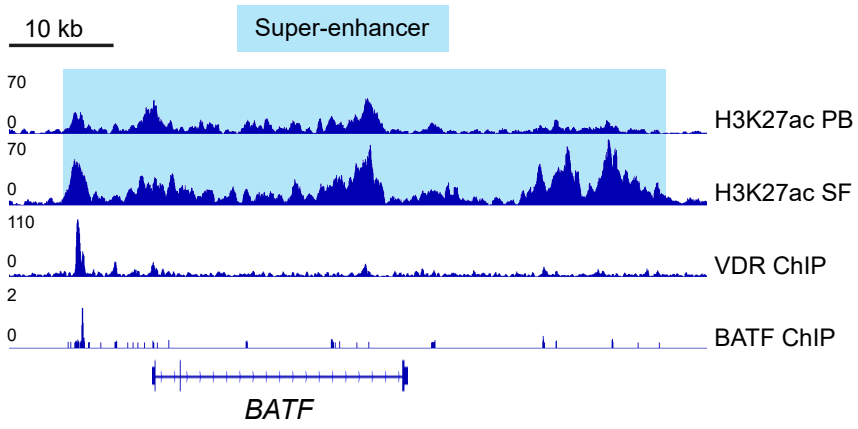


bioRxiv preprint doi: https://doi.org/10.1101/2020.09.30.319962; this version posted October 1 , 2020. The copyright holder for this preprint (which was not certified by peBreshictedik

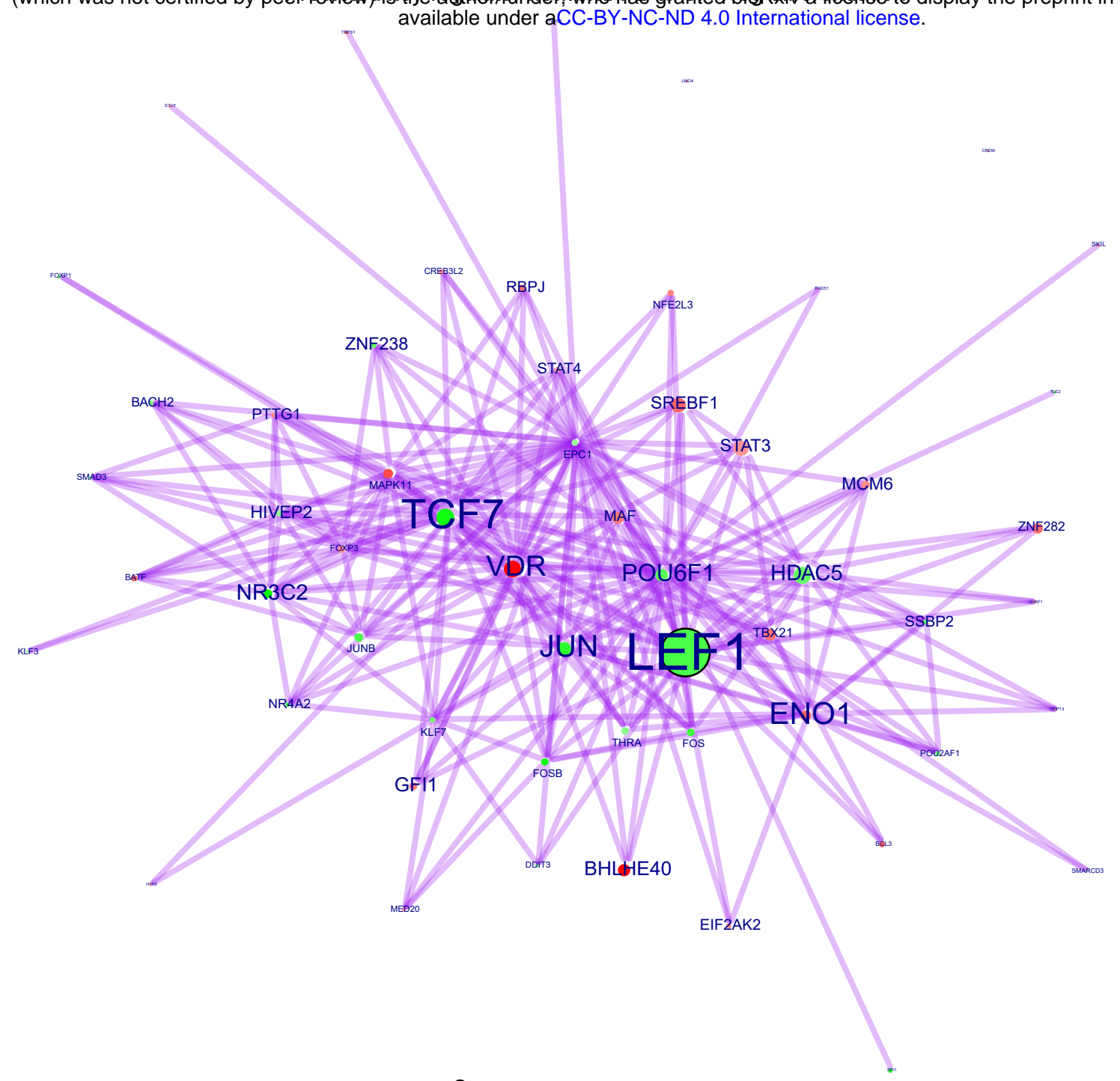

b

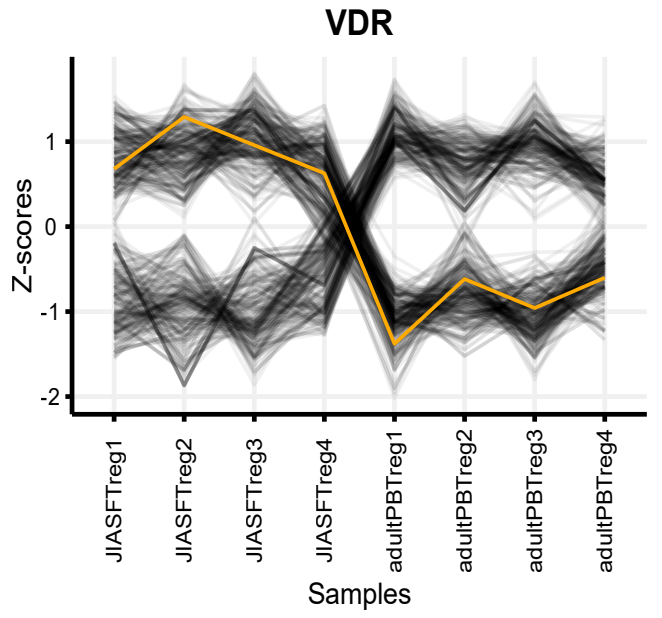

C

\begin{tabular}{ccc}
\multicolumn{3}{c}{ VDR-associated genes } \\
\hline POU6F1 & MAF & TCF3 \\
MLXIP & HSBP1 & JUNB \\
POLR1D & ZFPM1 & FOSB \\
ZBTB25 & SREBF1 & SCAF1 \\
MAX & PHF12 & TRIM28 \\
FOS & NF1 & ZNF341 \\
BCL11B & THRA & SLA2 \\
MTA1 & STAT3 & MAPK11 \\
ZNF174 & HDAC5 & FOXP3 \\
CREBBP & TBX21 & PIAS1 \\
SRCAP & SMURF2 & ZNF263
\end{tabular}

d

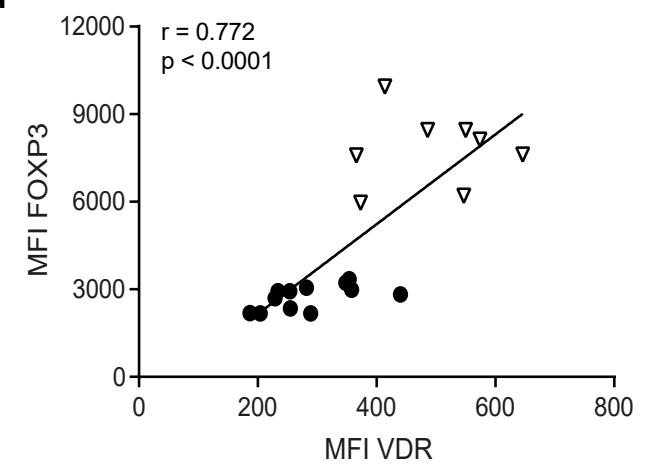

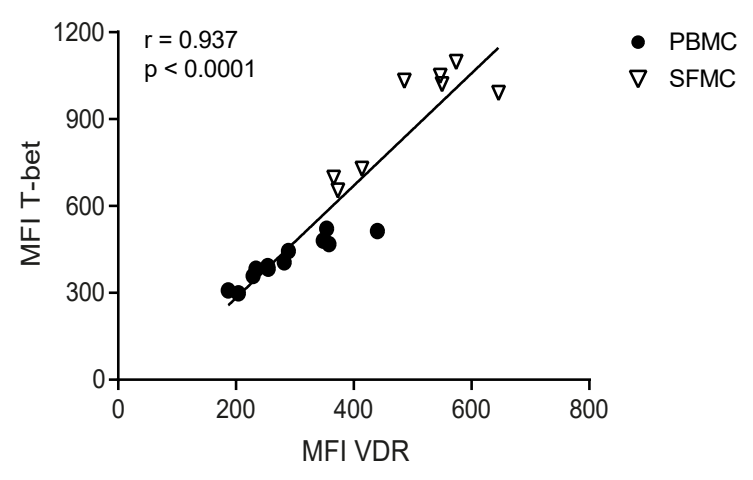




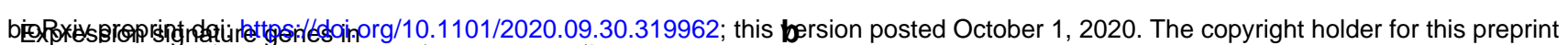

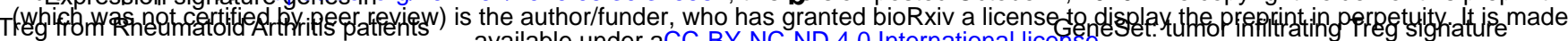

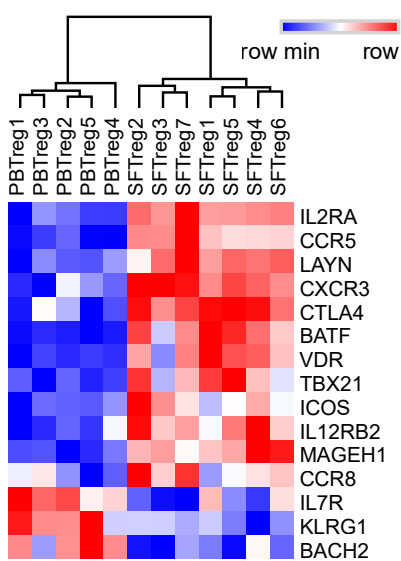

C
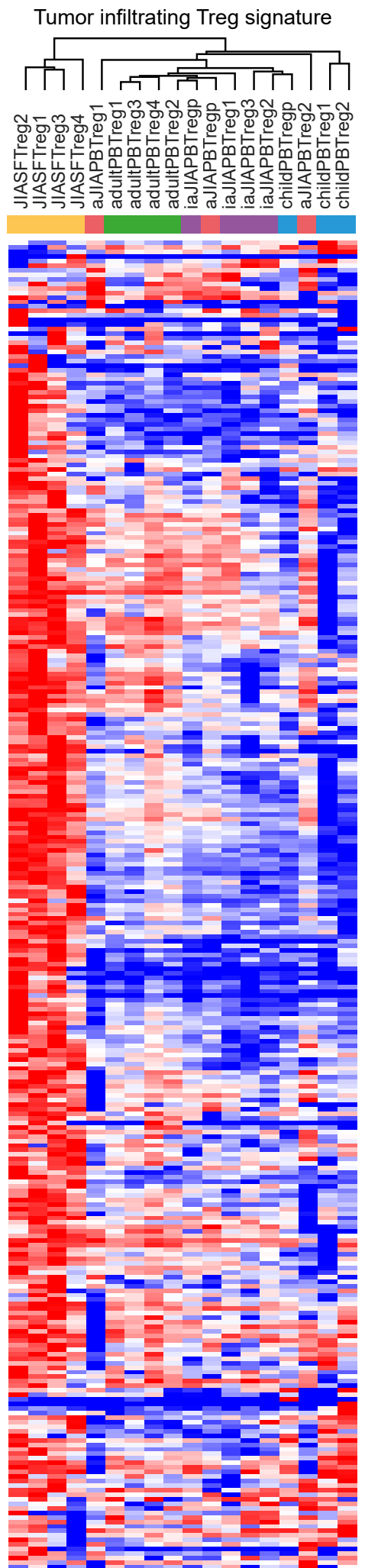

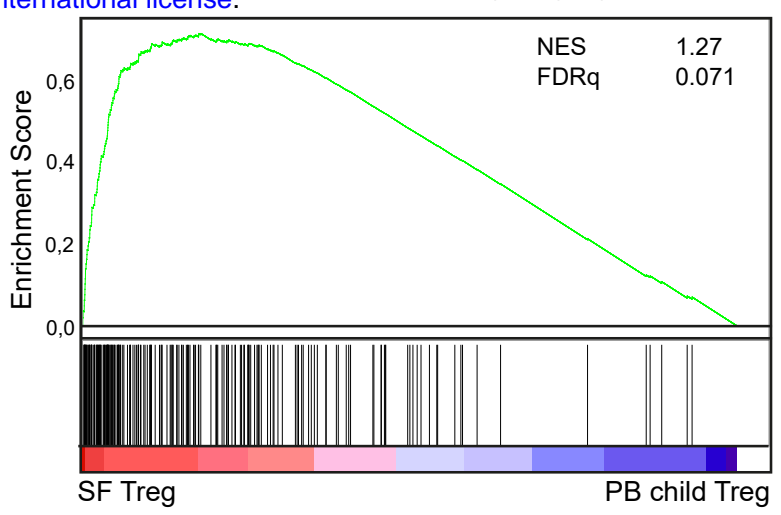

d

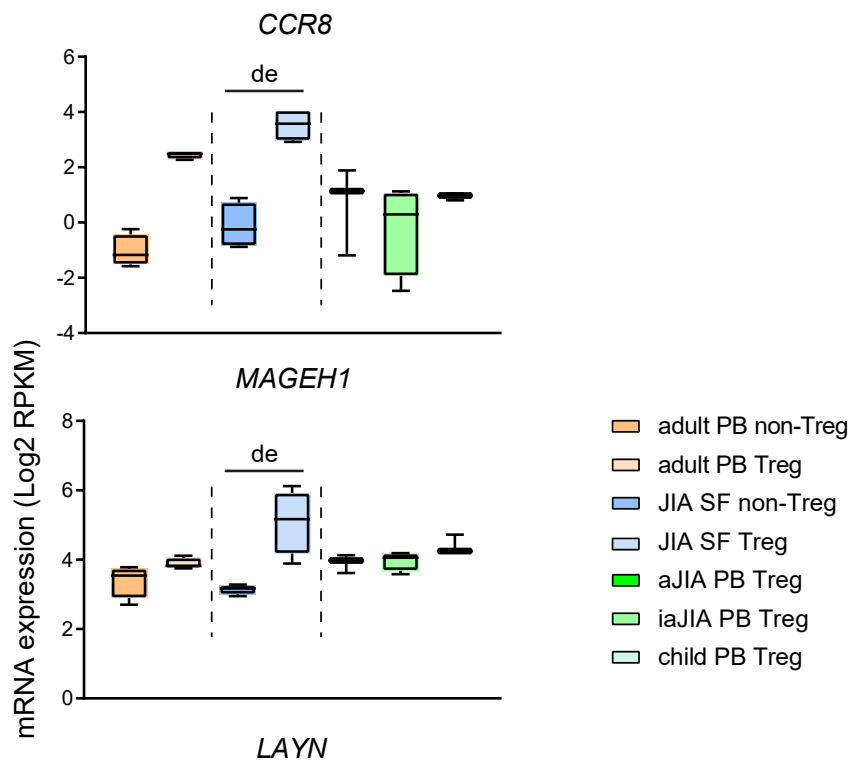

e
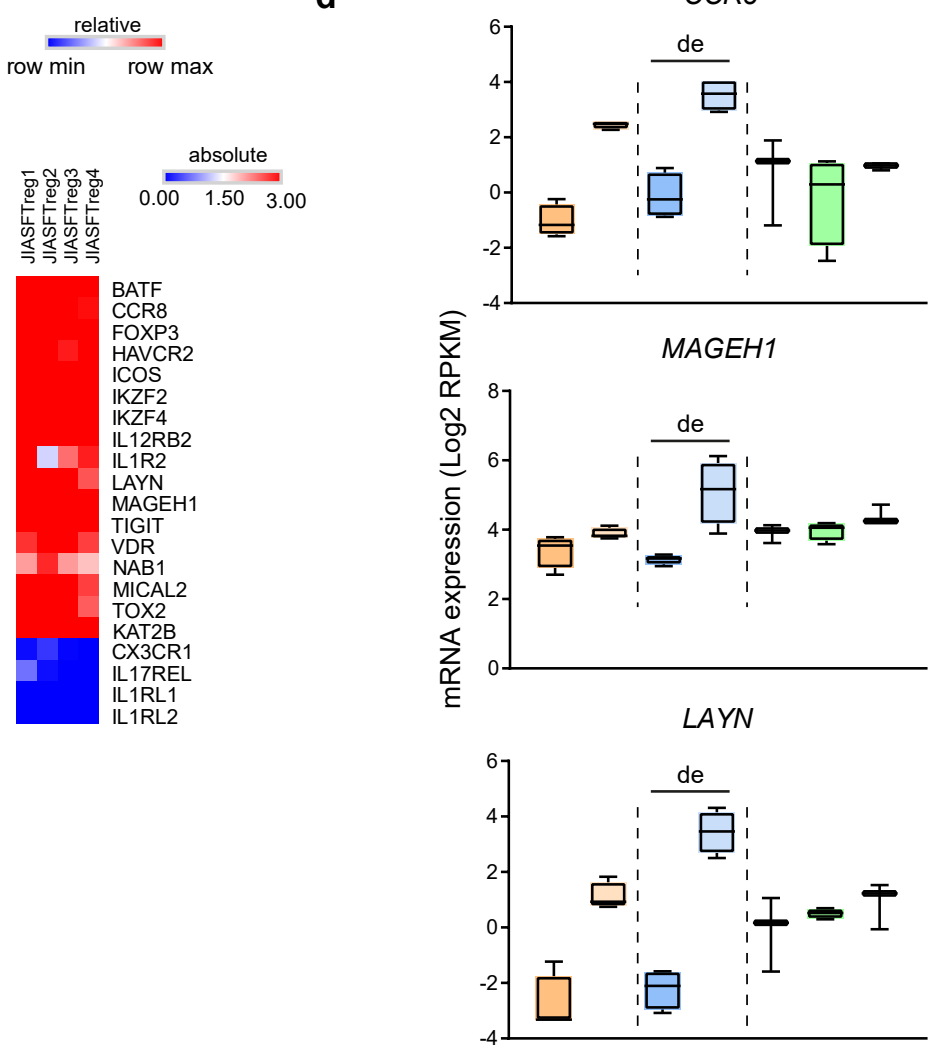

shared eTreg signature

\begin{tabular}{ccc}
\hline ARPP19 & HNRNPLL & PFKFB3 \\
BATF & ICOS & PHLDA1 \\
BCL2L1 & IL12RB2 & RBPJ \\
CCND2 & IL1R1 & SH2D2A \\
CCR8 & IL1R2 & SRGN \\
CD58 & KAT2B & TMEM173 \\
CTSC & LAIR2 & TNFRSF18 \\
ENTPD1 & LGALS3 & TNFRSF1B \\
FAM129A & MAF & TOX2 \\
GAPDH & MICAL2 & VDR \\
GCNT1 & NAMPT &
\end{tabular}

